\title{
Diffraction des neutrons : principe, dispositifs expérimentaux et applications
}

\author{
C. Muller
}

Laboratoire Matériaux et Microélectronique de Provence (L2MP), UMR 6137 du CNRS, Université de Toulon et du Var, BP. 132, 83957 La Garde cedex, France

\section{I- INTRODUCTION}

\section{II- PRINCIPES DE BASE DE LA DIFFRACTION DES NEUTRONS}

II-1. Généralités

II-2. Fonction de diffusion $\mathrm{S}(Q, \hbar \omega)$

III- INSTRUMENTS DE DIFFRACTION

III-1. Classification générale des instruments

III-2. Monochromatisation du faisceau

III-4. Filtres

IIII-5. Détection des neutrons

III-6. Fonction de résolution expérimentale

\section{IV- DIFFRACTION SUR MONOCRISTAL}

IV-1. Les diffractomètres

IV-2. A quelles informations structurales a-t-on accès?

IV-3. Stratégie de collecte et de traitement des données

IV-4. Comparaison de différents instruments

IV-5. Quelques exemples d'application

\section{V- DIFFRACTION SUR POUDRES}

V-1. Principe

V-2. Traitement des données de diffraction- Méthodes d'ajustement de profil

V-3. Comparaison de différents instruments

V-4. Quelques exemples d'applications

\section{VI- CONCLUSION}

\section{REFERENCES}

Résumé : La diffraction de neutrons, sur monocristal ou sur échantillon polycristallin (ou poudre), est une technique très largement utilisée, en science des matériaux comme en biologie, lorsque l'on souhaite déterminer la structure cristalline d'un composé ou d'une molécule. Toutefois, le degré de précision de la détermination structurale est très corrélé au choix de l'instrument utilisé. Il s'en suit que la question "comment choisir l'instrument le mieux adapté au composé et à la problématique ?" apparait comme fondamentale. L'objectif de ce cours est de tenter de répondre à cette question en décrivant brièvement les caractéristiques instrumentales de différents difftactomètres, en exposant les avantages spécifiques des expériences de diffraction de neutrons et en donnant quelques exemples d'application. 


\section{INTRODUCTION}

La diffraction de neutrons, sur monocristal ou sur échantillon polycristallin, est une technique très largement utilisée pour la détermination de structures cristallines dans des domaines allant de la science des matériaux à la biologie structurale. Toutefois, l'utilisation très coûteuse de sources de neutrons requiert une argumentation particulière qui doit mettre en avant les avantages spécifiques des expériences de diffraction de neutrons. Le point crucial est donc de savoir si la diffraction de neutrons, appliquée à une problématique définie, permettra ou non de fournir des informations pertinentes non accessibles par des techniques plus classiques et moins coûteuses telles que la diffraction de rayons $X$ sur source conventionnelle par exemple.

L'objectif de ce cours introductif aux techniques de diffraction neutronique est de tenter de répondre aux quelques questions suivantes :

- Quelles informations structurales peut-on extraire d'une expérience de diffraction de neutrons?

- Comment choisir l'instrument le mieux adapté au composé et/ou à la problématique ?

- Quelle est la résolution instrumentale nécessaire à la réalisation de l'expérience ?

- Quel type d'échantillon doit-on choisir : monocristal ou échantillon polycristallin?

\section{PRINCIPES DE BASE DE LA DIFFRACTION DES NEUTRONS}

\subsection{Généralités}

Le neutron est une particule neutre de spin $1 / 2$ qui interagit avec le noyau d'un atome et avec son moment magnétique lorsqu'il existe. Les caractéristiques générales du neutron peuvent être résumées de la manière suivante :

- L'absence d'interactions à longue distance assure une forte pénétration du neutron dans la matière (de l'ordre de quelques centimètres), le neutron étant alors très faiblement absorbé.

- Le neutron interagit avec le noyau des atomes et fournit ainsi une information complémentaire de celle donnée par les rayons $\mathrm{X}$ qui sont sensibles, quant à eux, au nuage électronique de l'atome. L'amplitude de l'interaction nucléaire dépend de la longueur de diffusion cohérente $b_{c o h}$ (ou longueur de Fermi) qui varie de manière non systématique d'un atome à l'autre ou d'un isotope à l'autre (Fig. 1). Cette particularité permet de déterminer précisément les positions atomiques d'éléments légers même s'ils sont en présence d'atomes plus lourds. Elle assure également la distinction entre deux atomes proches dans le tableau périodique ( $\mathrm{Mn}$ et $\mathrm{Fe}$ par exemple). Enfin, la substitution isotopique de l'hydrogène par le deutérium permet de localiser les sites hydrogénés dans les molécules organiques (polymères, molécules biologiques, ...).

- La forte interaction entre le spin 1/2 du neutron et le moment magnétique porté par un atome permet d'étudier l'ordre magnétique pouvant s'établir à basse température.

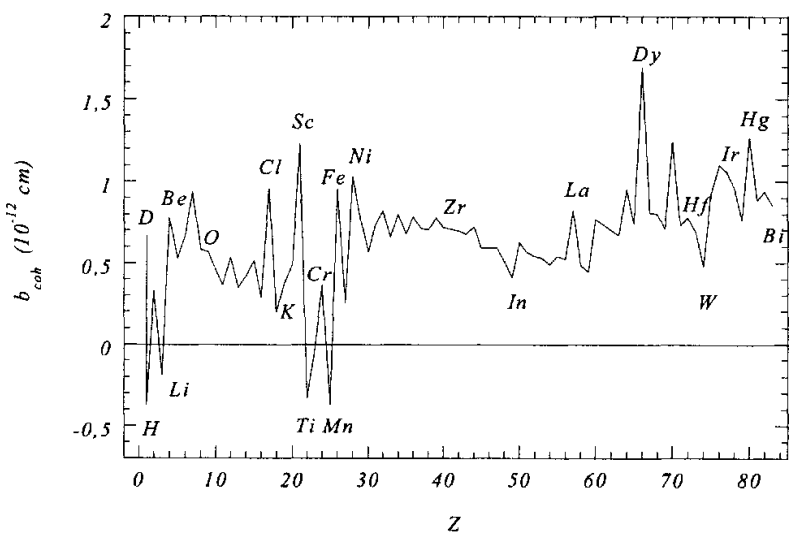

Figure 1: Variation de longueur de diffusion cohérente $b_{c o h}$ en fonction du numéro atomique $Z$ des éléments. 


\subsection{Fonction de diffusion $S(\vec{Q}, \hbar \omega)$}

Considérons un neutron arrivant avec un vecteur d'onde incident $\vec{k}_{\text {inc }}$. Ce neutron est diffusé par l'échantillon avec un vecteur d'onde sortant $\vec{k}_{\text {sor }}$ et le transfert de moment ou vecteur de diffusion $\vec{Q}$ résultant du processus de diffusion est défini par (Fig. 2):

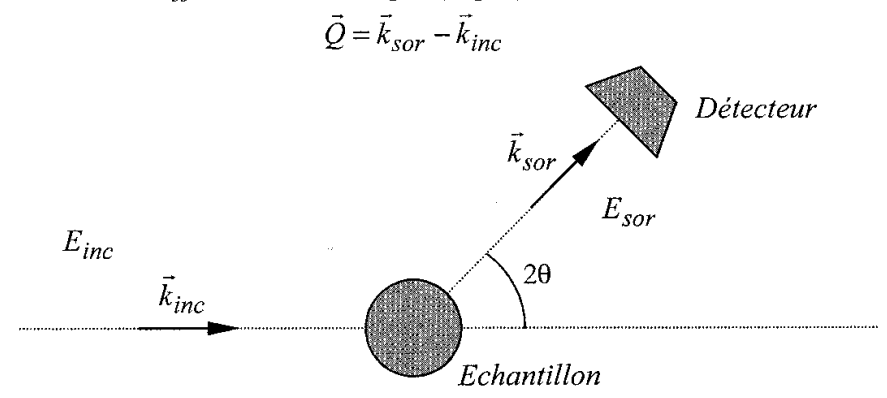

Figure 2: Processus de diffusion d'un neutron par un échantillon.

Au cours du processus de diffusion, le neutron change de direction, mais l'interaction se traduit également par un échange d'énergie $\hbar \omega$ (perte ou gain) entre le neutron et l'échantillon. La variation d'énergie $\hbar \omega$ est donnée par:

$$
\hbar \omega=E_{\text {sor }}-E_{\text {inc }}=\frac{\hbar^{2}}{2 m}\left(k_{\text {sor }}^{2}-k_{\text {inc }}^{2}\right)
$$

Par conséquent, réaliser une expérience de diffusion de neutrons revient à mesurer la fonction de diffusion notée $S(\vec{Q}, \hbar \omega)$ qui dépend des propriétés de l'échantillon. La diffusion des neutrons est donc une technique utilisée pour étudier la structure statique (structure moyenne dans le temps et dans l'espace) et la dynamique de la matière condensée.

Dans le cadre de ce cours, nous nous limiterons au cas de la diffraction qui correspond à la diffusion élastique de neutrons $(\hbar \omega=0)$. La dépendance en $\vec{Q}$ de la fonction de diffusion $S(\vec{Q}, \hbar \omega=0)$ donne alors une information directe sur l'arrangement spatial tridimensionnel $\rho(\vec{r})$ des atomes dans la maille et permet ainsi de déterminer la structure cristalline de l'échantillon. Pour un cristal périodique parfait, la fonction de diffusion $S(\vec{Q}, \hbar \omega=0)$ n'est non nulle que pour certaines valeurs du vecteur de diffusion respectant la condition dite de Bragg.

En diffraction, le transfert d'énergie étant nul ( $\hbar \omega=0$ ), le vecteur de diffusion $\vec{Q}$ ne dépend plus que de la valeur du vecteur incident $k_{\text {inc }}$ (ou de la longueur d'onde $\lambda$ ) et de l'angle de diffusion $2 \theta$ (cf. Fig. 2) :

$$
\left\|\vec{k}_{\text {inc }}\right\|=\left\|\vec{k}_{\text {sor }}\right\|=\frac{2 \pi}{\lambda} \text { et } Q=2 \cdot k_{\text {inc }} \cdot \sin \theta=4 \pi \frac{\sin \theta}{\lambda}
$$

\section{INSTRUMENTS DE DIFFRACTION}

\subsection{Classification générale des instruments}

Les instruments utilisés en diffusion de neutrons sont classés en deux catégories : les diffractomètres et les spectromètres. Les diffractomètres, dédiés à l'étude de la dépendance en $\vec{Q}$ de la fonction de diffusion $S(\vec{Q})$ à transfert d'énergie nul ( $\hbar \omega=0)$, sont utilisés pour déterminer la structure cristallographique d'un échantillon. On parle en général de diffractomètres 2-axes. Les spectromètres, permettant d'effectuer une analyse simultanée en énergie et en transfert de moment de la fonction de diffusion $S(\vec{Q}, \hbar \omega)$, assurent la compréhension des phénomènes dynamiques pouvant apparaître dans l'échantillon (mouvements coopératifs, mouvements diffusifs, réorientations...). 
Dans la famille des diffractomètres, on distingue à nouveau deux catégories selon le type d'expérience réalisée (Fig. 2) :

- La première méthode consiste à fixer le vecteur d'onde incident $k_{i n c}$ (rayonnement monochromatique) et à faire varier l'angle de diffraction $2 \theta$ (méthode classique en diffraction de rayons $\mathrm{X}$ ) ;

- La seconde méthode consiste à fixer l'angle de diffraction $2 \theta$ et à faire varier le vecteur d'onde incident $k_{i n c}$ (faisceau polychromatique).

La première méthode, nécessitant un dispositif de monochromatisation, est généralement utilisée sur un réacteur qui délivre un flux continu de neutrons. La seconde méthode est essentiellement utilisée sur les sources à spallation qui délivrent des "pulses" de neutrons et pour lesquelles la mesure du temps de vol du neutron (TOF = Time Of Flight) permet de déterminer le vecteur d'onde incident $k_{\text {inc }}$.

Dans le cadre de ce cours, nous allons considérer que les neutrons utilisés lors d'une expérience de diffraction sont produits par un réacteur délivrant un flux continu de neutrons : les neutrons, issus de la fission de noyaux lourds et thermalisés par le modérateur, forment un faisceau divergent ayant une certaine distribution continue de longueurs d'onde. Une expérience de diffraction nécessitant un faisceau parallèle monochromatique, les neutrons doivent donc être monochromatisés et collimatés avant d'atteindre l'échantillon. La dispersion en longueur d'onde du faisceau incident, sa divergence, la largeur des fentes, la mosaïcité du monochromateur... sont autant de paramètres qui vont déterminer la résolution de l'instrument.

\subsection{Monochromatisation du faisceau}

\subsubsection{Monochromateurs monocristallins}

Un monochromateur est un monocristal qui permet de sélectionner, par réflexion de Bragg, un faisceau de neutrons monochromatiques. Si un faisceau de neutrons présentant une distribution continue de longueurs d'onde arrive sur un monocristal, plusieurs familles de plans réticulaires d'indices de Miller (hkl) donnent lieu à des réflexions des Bragg dans des directions bien définies, chaque réflexion étant associée à une longueur d'onde vérifiant la loi de Bragg :

$$
\text { 2.d. } \sin \theta_{m}=\lambda
$$

où $\lambda$ est la longueur d'onde et $d$ la distance inter-réticulaire.

En ajustant l'orientation du monochromateur par rapport à la direction du faisceau incident, il est alors possible de collecter, dans une direction de l'espace, un faisceau de neutrons monochromatiques (Fig. 3). L'angle entre le faisceau incident et le faisceau diffracté est appelé angle de "take-off" du monochromateur (ou angle de coupure). Comme nous le verrons dans le paragraphe 3.3, cet angle est important puisqu'il détermine en partie la résolution instrumentale du diffractomètre.

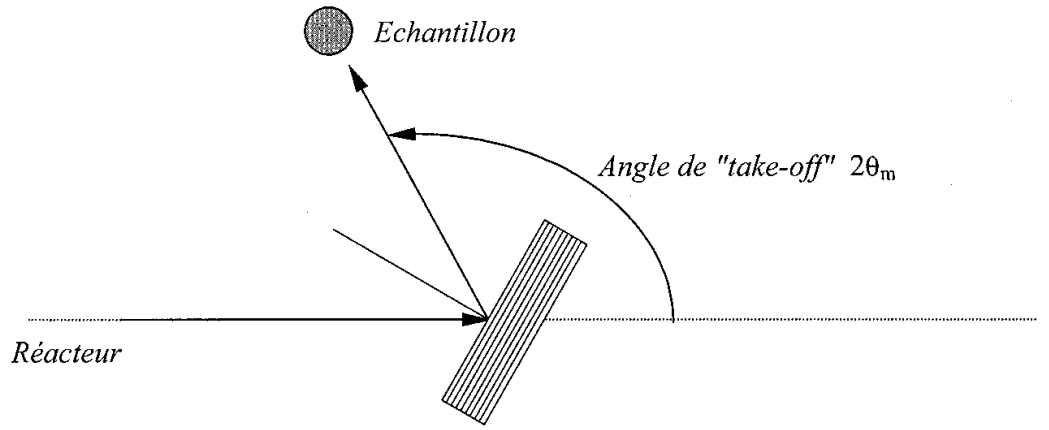

Figure 3: Principe d'un monochromateur monocristallin. 
En pratique, un monochromateur est souvent constitué de plusieurs monocristaux de silicium, de germanium, de cuivre, ou de graphite pyrolitique, l'ensemble faisant une vingtaine de centimètres de haut.

Le pouvoir réflecteur d'un monocristal parfait dépend bien sûr du facteur de structure de la réflexion considérée mais également de la mosaïcité du cristal qui correspond au degré de désorientation des cristallites autour d'une orientation moyenne. Cette mosaïque est quantifiée par le paramètre $\beta_{m}$ défini comme la largeur intégrale du monochromateur. Cette largeur intégrale est déduite d'une "rocking curve" du monochromateur.

Lorsque la mosaïcité du monochromateur est faible (cas d'un cristal parfait), le faisceau subit une forte extinction par réflexion multiple à l'intérieur du cristal (extinction primaire). Une façon d'augmenter la réflectivité du monochromateur est d'augmenter, de manière contrôlée, la mosaïcité du cristal. Toutefois, une mosaïcité plus grande entraîne une diminution de la monochromaticité du faisceau et un élargissement angulaire. Le compromis habituel consiste à utiliser des monocristaux dont la mosaïcité est de l'ordre d'une vingtaine de minutes d'arc.

Le faisceau incident étant souvent très large, il est possible d'associer les cristaux de manière à réaliser un monochromateur focalisant qui permet de concentrer le faisceau et d'augmenter ainsi le flux au niveau de l'échantillon [1,2]. Le montage, schématisé sur la Figure 4 consiste en un assemblage de cristaux à orientation modulable permettant de faire varier la courbure de l'ensemble par une action mécanique. Ce dispositif mettant en jeu à la fois la réflexion d'une onde par une surface courbe et la réflexion de Bragg, les conditions de focalisation doivent être ajustées pour chaque longueur d'onde.

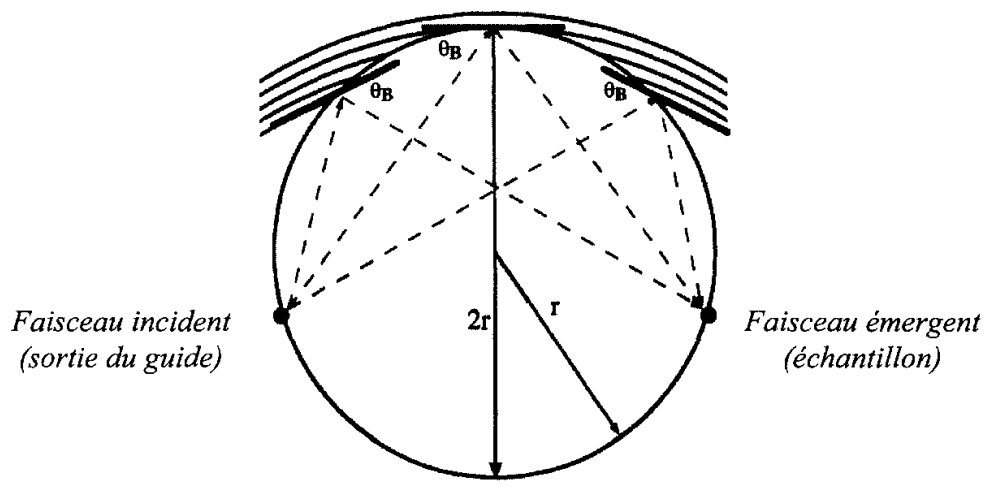

Figure 4: Principe d'un monochromateur focalisant [1].

\subsubsection{Miroirs multicouches}

Une alternative possible à l'utilisation de monocristaux comme monochromateurs est l'utilisation de miroirs multicouches. Dans ce cas, le dispositif réfléchissant est constitué d'une alternance de couches minces de deux matériaux ( $\mathrm{Mn}$ et Ge par exemple) déposées sur un substrat (verre ou silicium) et formant ainsi un système périodique dans la direction normale au plan des couches, la périodicité étant déterminée par l'épaisseur des couches. Cet empilement régulier de couches minces donne lieu à des réflexions de Bragg et permet ainsi son utilisation comme monochromateur. En choisissant de manière appropriée les matériaux de l'empilement, on obtient des multicouches très réflectrices ayant une très faible contamination par les harmoniques (cf. §3.4).

\subsubsection{Sélecteurs de vitesse}

L'utilisation d'un monocristal comme monochromateur est limitée à une longueur d'onde maximale compatible avec la loi de Bragg (environ $6 \AA$ pour un monochromateur graphite). Pour monochromatiser des longueurs d'onde supérieures à $6 \AA$, on utilise alors des méthodes mécaniques 
pour sélectionner les neutrons en fonction de leur vitesse : la Figure 5 présente le schéma de principe d'un sélecteur de vitesse.

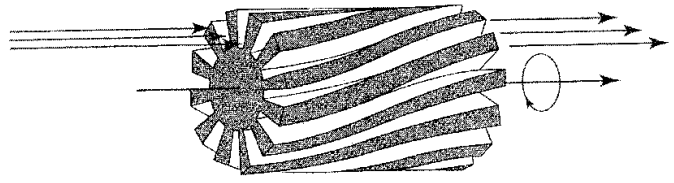

Figure 5: Principe du sélecteur de vitesse.

Sur un cylindre absorbant, on grave des sillons hélicoïdaux avec un pas d'hélice noté $p$. Si le cylindre tourne autour de son axe de révolution avec une vitesse angulaire $\omega$, chaque sillon ne laisse passer que les neutrons ayant une vitesse $v=p \frac{\omega}{2 \pi}$. Ce dispositif permet ainsi d'obtenir un flux continu de neutrons monochromatiques. Toutefois, la sélectivité en vitesse n'excédant pas 10 à $20 \%$, ce type de monochromateur n'est utilisé que sur des instruments ne nécessitant pas une très bonne résolution en longueur d'onde.

\subsubsection{Choppers}

La seconde méthode mécanique pour produire des neutrons monochromatiques consiste à utiliser des choppers ("hacheurs"). Comparativement au sélecteur de vitesse, un chopper ne délivre pas un flux contimu de neutrons mais des pulses monochromatiques.

Le dispositif de base consiste à placer sur la trajectoire du faisceau plusieurs disques recouverts d'une couche absorbante (d'oxyde de gadolinium $\mathrm{Gd}_{2} \mathrm{O}_{3}$ par exemple) sur lesquels sont ménagées des fenêtres transparentes. Considérons à titre d'exemple le monochromateur à deux disques présenté sur la Figure $6:$ les deux disques séparés par une distance $L$ tournent dans le même sens à la même vitesse angulaire $\omega$, mais présentent entre eux un angle de déphasage $\varphi$.

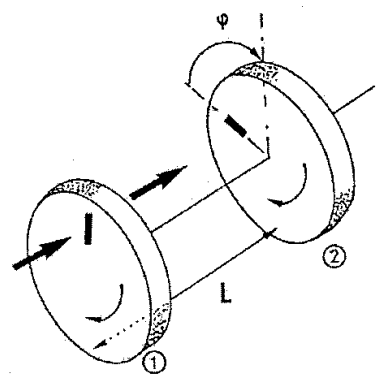

Figure 6: Utilisation de "choppers" pour la monochromatisation de neutrons.

Le premier disque a pour rôle de produire des pulses de neutrons polychromatiques et le second permet de sélectionner des neutrons monochromatiques. Seuls les neutrons ayant une vitesse $\nu=L \frac{\omega}{\varphi}$ sont transmis. La longueur d'onde du faisceau de neutrons dépend ainsi de la distance séparant les deux disques et de leur vitesse de rotation.

Ce dispositif de monochromatisation est très largement utilisé sur les spectromètres temps de vol dédiés aux expériences de diffusion inélastique et quasiélastique de neutrons. Toutefois, il se trouve également sur des instruments de diffraction sur échantillons polycristallins utilisant un flux de neutrons produits par une source à spallation (cf. §5.4.4).

A titre dillustration, la Figure 7 présente le chopper qui sera installé sur la source à spallation KFKI de Budapest [3]. L'instrument utilisant ces choppers pour générer des pulses de neutrons peut être installé indifféremment sur un réacteur à haut flux ou sur une source à spallation. 


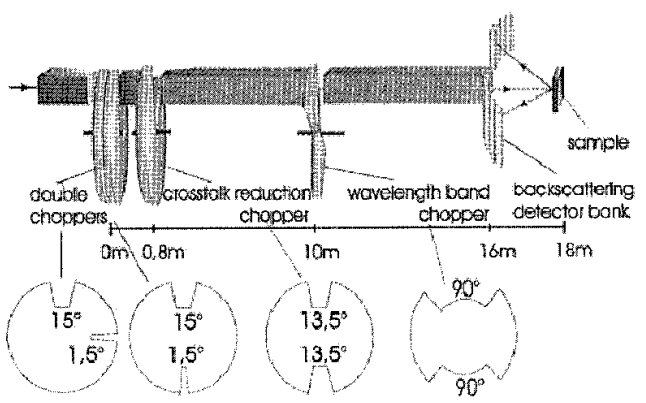

Figure 7: Choppers développés sur la source à spallation KFKI [3].

Dans le cas présent, les disques 1 et 2 fonctionnent de la manière décrite précédemment, la bande de longueur d'onde transmise $\Delta \lambda$ dépendant de la distance entre les deux disques (variant de 20 à $60 \mathrm{~mm}$ ). Les choppers 3 et 4 ont pour rôles essentiels de limiter le nombre de pulses et d'éviter la contamination des harmoniques.

\subsection{Collimation du faisceau}

La réalisation d'une expérience de diffraction requiert un faisceau parallèle obtenu par collimation des neutrons monochromatiques. Les collimateurs conventionnels (ou fentes de Soller), dont le rôle est de limiter la divergence du faisceau avant et après le monochromateur, sont formés d'un ensemble de lames parallèles faites d'un matériau absorbant les neutrons. Les neutrons arrivant sur le collimateur avec un angle supérieur à l'angle d'ouverture sont alors absorbés.

En général, les qualités requises pour un collimateur sont une transmission élevée et un profil bien défini dont l'effet sur la résolution instrumentale peut être pris en compte. De plus, ces collimateurs doivent être robustes et facilement mis en forme dans des dimensions adaptées à la taille du faisceau incident. La plupart des collimateurs modernes, constitués de lames de mylar recouvertes d'oxyde de gadolinium $\mathrm{Gd}_{2} \mathrm{O}_{3}$, atteignent une transmission de l'ordre de $95 \%$ [4]. D'autres collimateurs à parois réflectrices sont développés actuellement afin d'augmenter la transmission sans diminuer la résolution angulaire. A titre d'exemple, des tests sont menés sur des wafers de silicium recouverts sur d'une couche réflectrice (alliage $\mathrm{Fe}_{89} \mathrm{Co}_{11}$ ) elle-même recouverte d'une couche absorbante de gadolinium [5].

\subsection{Filtres}

Un monochromateur donne, pour une longueur d'onde $\lambda$, une réflexion de Bragg à un angle $2 \theta_{m}$ tel que la loi de Bragg soit vérifiée. Cependant, cette condition de diffraction n'est pas seulement réalisée pour la longueur d'onde $\lambda$, mais elle l'est aussi pour les harmoniques d'ordres supérieurs $\frac{\lambda}{2}, \frac{\lambda}{3}, \ldots \frac{\lambda}{n}$ qui contaminent ainsi le faisceau monochromatique.

Pour éviter cette contamination du faisceau, on utilise des filtres permettant l'élimination des harmoniques. Les filtres les plus courants sont en graphite pyrolitique polycristallin très fortement texturé dans la direction de l'axe hexagonal $c$, l'axe $a$ étant complètement désorienté (texture de fibre). Si l'axe $c$ est aligné avec la direction du faisceau incident, ce filtre est alors transparent pour les longueurs d'onde élevées et opaque pour les plus courtes. L'utilisation de filtres de graphite est bien adaptée à des longueurs d'onde comprises entre 2,2 et $4 \AA$ environ. Pour des longueurs d'onde supérieures à $4 \AA$, des filtres en béryllium sont alors préférés. Un dispositif de refroidissement du filtre à la température de l'azote liquide est souvent mis en place afin d'augmenter la transmission du faisceau primaire (sans toutefois augmenter la transmission des harmoniques). 


\subsection{Détection des neutrons}

\subsubsection{Détecteurs à scintillation}

Dans un détecteur à scintillation, un neutron est absorbé par un plastique ou un verre chargé en isotopes ${ }^{6} \mathrm{Li}$ et en $\mathrm{ZnS}(\mathrm{Ag})$. L'absorption du neutron se manifeste par l'excitation des atomes du verre qui, en se désexcitant, émettent des photons guidés vers un photomultiplicateur. Avec une électronique rapide et de larges scintillateurs, on peut couvrir de grandes surfaces avec un taux de comptage élevé.

Il faut signaler que des détecteurs à base de silicium [6,7], très utilisés en physique des hautes énergies comme traceurs de particules, sont actuellement développés en détection neutronique pour leur grande résolution spatiale à des taux de comptage élevés.

\subsubsection{Détecteurs proportionnels à gaz}

Le principe de fonctionnement d'un détecteur proportionnel à gaz est basé sur l'absorption d'un neutron par un gaz sous pression $\left({ }^{3} \mathrm{He}, \mathrm{BF}_{3}\right.$ ou $\mathrm{CF}_{4}$ enrichi en $\left.{ }^{10} \mathrm{~B}\right)$. L'absorption d'un neutron produit des paires ion-électron dans le gaz. Une anode sous haute tension permet non seulement d'éviter les recombinaisons des paires, mais accélère aussi les électrons qui ionisent, à leur tour, d'autres atomes du gaz créant ainsi un phénomène d'avalanche. Ce phénomène se manifeste par une décharge sur l'anode. Dans ce type de dispositif, le taux de comptage est limité par le temps de collection des ions et par la rapidité de l'électronique associée.

La densité du gaz absorbant et l'épaisseur du détecteur sont adaptées pour obtenir une probabilité de détection du neutron voisine de $100 \%$. Le principal avantage des détecteurs à gaz est leur grande efficacité pour la détection des neutrons thermiques. Ce type de détecteur est souvent utilisé comme moniteur, i.e. détecteur placé avant l'échantillon et servant à compter le nombre de neutrons incidents.

\subsubsection{Multidétecteurs}

Les neutrons étant diffusés dans toutes les directions de l'espace, il est très intéressant d'utiliser des multidétecteurs capables de couvrir de grands angles solides. La manière la plus simple d'obtenir un multidétecteur est d'associer plusieurs détecteurs à gaz individuels tels que ceux décrits dans le paragraphe précédent. Ce type de détecteur est très utilisé en diffraction sur poudres. A titre d'exemple, la Figure 8 présente le multidétecteur installé sur l'instrument $3 \mathrm{~T} 2$ au Laboratoire Léon Brillouin (LLB, CE - Saclay) qui est constitué de 20 compteurs à gaz ${ }^{3} \mathrm{He}$ espacés de $3^{\circ}$ et couvrant $57^{\circ}$.

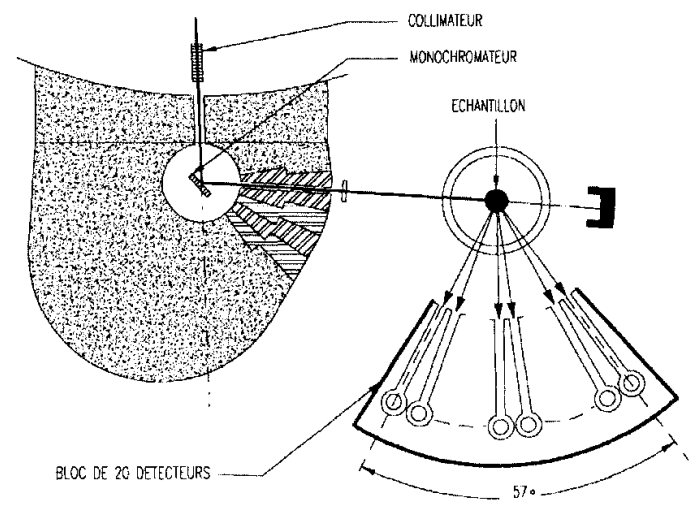

Figure 8: Caractéristiques instrumentales du diffractomètre sur poudres 3T2 (LLB).

De manière à améliorer la résolution angulaire, à augmenter l'efficacité du détecteur et à réduire les coûts, on peut également utiliser un seul détecteur constitué de plusieurs cellules de détection. Ce détecteur porte généralement le nom de PSD ("Position Sensitive Detector"). A titre d'exemple, le multidétecteur installé sur l'instrument de diffraction sur poudres D20 à l'Institut Laue Langevin 
(ILL, Grenoble) est constitué de 1600 cellules couvrant $160^{\circ}$ (soit 10 cellules par degré) et son rayon de courbure est de $1,5 \mathrm{~m}$ [8,9]. Le détecteur est rempli d'un mélange de 3,1 bars de ${ }^{3} \mathrm{He}$ et 0,8 bar de $\mathrm{CF}_{4}$.

Sur la source à spallation Suisse SINQ, Fisher et al. ont également développé, pour un instrument de diffraction sur poudres haute résolution, un multidétecteur à ${ }^{3} \mathrm{He}$ de 1600 cellules avec une séparation angulaire de $0,1^{\circ}[10]$.

\subsubsection{Détecteurs bidimensionnels}

En diffusion aux petits angles (études de grandes structures cristallines) et en diffraction sur monocristaux, il est courant d'utiliser des détecteurs bidimensionnels. La Figure 9 présente le principe d'un détecteur proportionnel "multi-fils", dans lequel l'anode, formée d'une série de fils individuels parallèles (diamètre de l'ordre de $20 \mu \mathrm{m}$ ), se trouve placée à mi-distance entre deux cathodes formant deux réseaux perpendiculaires de fils parallèles. Les électrons crées lors du processus d'absorption du neutron sont collectés sur l'anode et une charge miroir est générée sur les deux cathodes qui délivrent ainsi un signal en $\mathrm{X}$ et en $\mathrm{Y}$ permettant de localiser le neutron incident.

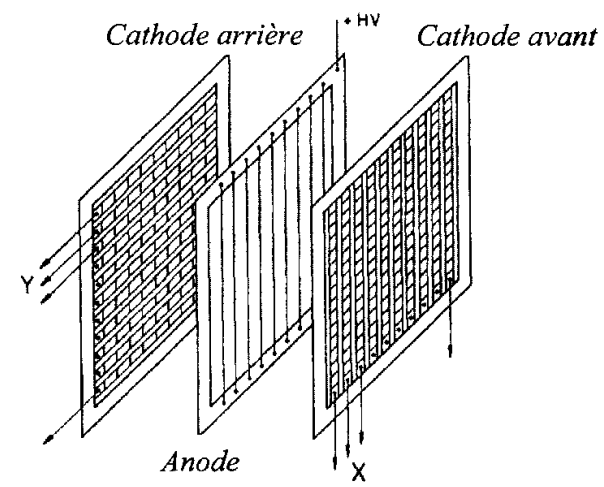

Figure 9: Principe d'un détecteur bidimensionnel à gaz.

A titre d'exemple, ce type de détecteur a été développé à GeNF en Allemagne (Geesthacht Neutron Facility) sur le nouveau diffractomètre ARES dédié aux analyses de contraintes [11]. Il s'agit d'un détecteur à mélange ${ }^{3} \mathrm{He}-\mathrm{CF}_{4}$ couvrant une surface de $300 \mathrm{~mm} \times 300 \mathrm{~mm}$, avec une efficacité de détection élevée pour des longueurs d'onde supérieurs à $1,5 \AA$ et une résolution spatiale de $2 \mathrm{~mm} \times 2 \mathrm{~mm}$.

Outre les détecteurs bidimensionnels à gaz, il existe également des détecteurs bidimensionnels appelés "image plate". La Figure 10 présente la photo du diffractomètre Laue LADI à l'ILL [12].

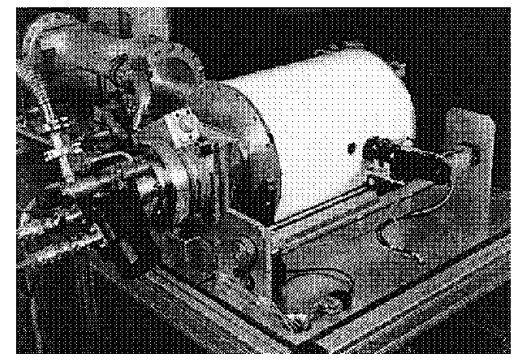

Figure 10: "Image plate" installée sur le diffractomètre Laue LADI (ILL) [12].

Le dispositif de détection utilise une "image plate" constituée d'un liant organique chargé en $\mathrm{BaFBr}$ dopé à l'europium $\left(\mathrm{Eu}^{2+}\right)$ servant de matériau photostimulable et en oxyde de gadolinium 
$\mathrm{Gd}_{2} \mathrm{O}_{3}$ assurant la "conversion" des neutrons en électrons et photons $\gamma$ rapides. Cette "image plate" couvrant une surface de $800 \mathrm{~mm} \times 400 \mathrm{~mm}$, est placée sur la surface extérieure d'un cylindre d'aluminium de $4 \mathrm{~mm}$ d'épaisseur et de $1000 \mathrm{~mm}$ de circonférence. Un trou ménagé sur le cylindre permet d'amener les neutrons jusqu'au cristal placé au centre du cylindre. L'image plate est ensuite révélée par un faisceau laser émettant dans le rouge.

Pour plus de détails sur les détecteurs bidimensionnels, on pourra se référer à un article de revue paru récemment dans Nuclear Instruments and Methods in Physics Research [13] et au travail de Takasaki et al. qui ont développé une "image plate" bien adaptée à la cristallographie des macromolécules biologiques [14].

\subsection{Fonction de résolution instrumentale}

\subsubsection{Profil d'une raie de diffraction}

De façon générale, le profil expérimental $h(x)$ d'une raie de diffraction est le produit de convolution d'une fonction de résolution instrumentale $f(x)$ et du profil lié à la microstructure de l'échantillon $g(x)$ :

$$
h(x)=f(x) \otimes g(x)=\int_{-\infty}^{+\infty} f(y) \cdot g(x-y) \cdot d y
$$

En diffraction de neutrons, la résolution instrumentale l'emporte souvent sur la résolution liée à la microstructure de l'échantillon (taille de cristallites et microdéformations). En relation avec ce qui a été décrit dans les paragraphes précédents, il apparaît que la résolution instrumentale d'un diffractomètre de neutrons est limitée par des paramètres tels que :

- la dispersion en longueur d'onde de la source ;

- la largeur des fentes et l'ouverture angulaire du collimateur ;

- la mosaïcité du monochromateur ;

- le bruit de l'électronique de mesure (détecteurs)....

De ce fait, en diffraction neutronique, le profil des raies est souvent considéré comme gaussien et l'évolution angulaire de la largeur à mi-hauteur des raies suit la fonction proposée par Caglioti et al. qui va être décrite dans le paragraphe suivant [15].

\subsubsection{Fonction de résolution instrumentale}

Dans les expériences de diffraction de neutrons, les collimateurs sont placés comme indiqué sur la Figure 11: $\alpha_{1}, \alpha_{2}$ et $\alpha_{3}$ représentent respectivement la divergence angulaire horizontale des trois collimateurs et $\beta_{m}$ est la largeur intégrale du monochromateur liée à sa mosaïcité (cf. §3.2).

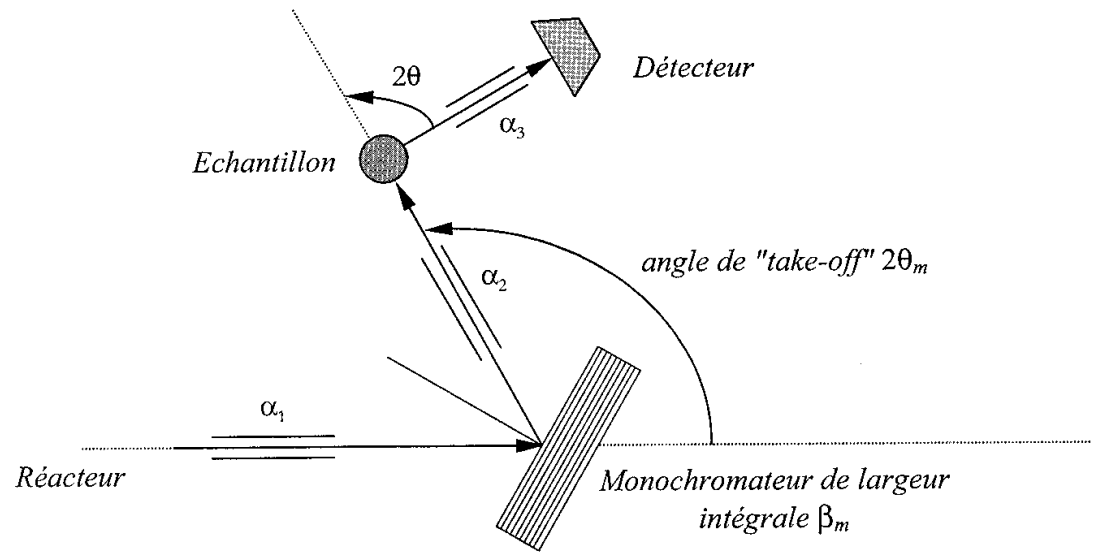

Figure 11: Disposition des collimateurs sur un diffractomètre de neutrons. 
Dans les années 1960, Caglioti et al. ont déterminé l'expression de la largeur à mi-hauteur $\mathrm{H}$ d'une réflexion de Bragg (profil supposé gaussien) en fonction de la divergence angulaire horizontale des trois collimateurs [15]:

$$
H=\sqrt{\frac{\alpha_{1}^{2} \alpha_{2}^{2}+\alpha_{1}^{2} \alpha_{3}^{2}+\alpha_{2}^{2} \alpha_{3}^{2}+4 \beta_{m}^{2}\left(\alpha_{2}^{2}+\alpha_{3}^{2}\right)-4 \frac{\tan \theta}{\tan \theta_{m}} \alpha_{2}^{2}\left(\alpha_{1}^{2}+2 \beta_{m}^{2}\right)+4 \frac{\tan ^{2} \theta}{\tan ^{2} \theta_{m}}\left(\alpha_{1}^{2} \alpha_{2}^{2}+\alpha_{1}^{2} \beta_{m}^{2}+\alpha_{2}^{2} \beta_{m}^{2}\right)}{\alpha_{1}^{2}+\alpha_{2}^{2}+4 \beta_{m}^{2}}}
$$

La largeur à mi-hauteur minimale, correspondant au maximum de résolution du diffractomètre, est obtenue lorsque $\theta \approx \theta_{m}$ et vaut :

$$
H \approx \sqrt{\alpha_{1}^{2}+\alpha_{\frac{2}{3}}^{2}}
$$

Il en résulte qu'un diffractomètre de haute résolution instrumentale doit avoir [16] :

- un angle de "take-off" élevé $\left(2 \theta_{m}>120^{\circ}\right)$ pour placer le maximum de résolution dans le domaine angulaire présentant une grande densité de réflexions de Bragg ;

- des fentes de Soller étroites avant le monochromateur et entre l'échantillon et le détecteur (cf. Fig. 11).

Pratiquement, la fonction de résolution instrumentale des diffractomètres de neutrons est donnée par la fonction dite de Caglioti qui se met sous la forme [15] :

$$
H=\sqrt{U \cdot \tan ^{2} \theta+V \cdot \tan \theta+W}
$$

où les coefficients $U, V$ et $W$ dépendent des collimations $\alpha_{1}, \alpha_{2}$ et $\alpha_{3}$, et de la mosaïcité $\beta_{m}$ du monochromateur.

La Figure 12 présente le schéma de principe du diffractomètre sur poudres haute résolution D2B de l'ILL (on notera en particulier la valeur élevée de l'angle de "take-off").

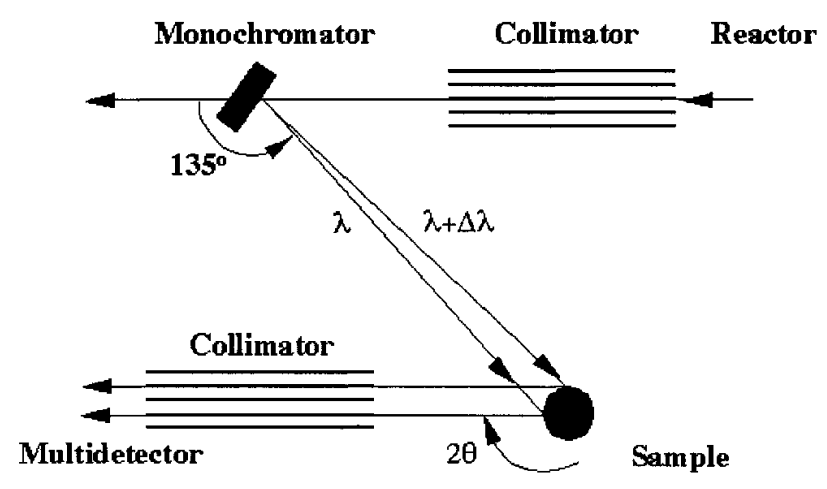

Figure 12: Schéma de principe du diffractomètre haute résolution D2B (ILL).

A titre d'illustration, la Figure 13 compare les résolutions instrumentales de différents diffractomètres sur poudres : D1A et D1B (ILL), 3T2 (LLB) et D5000 (diffractomètre de rayons X de Bruker-Siemens). 


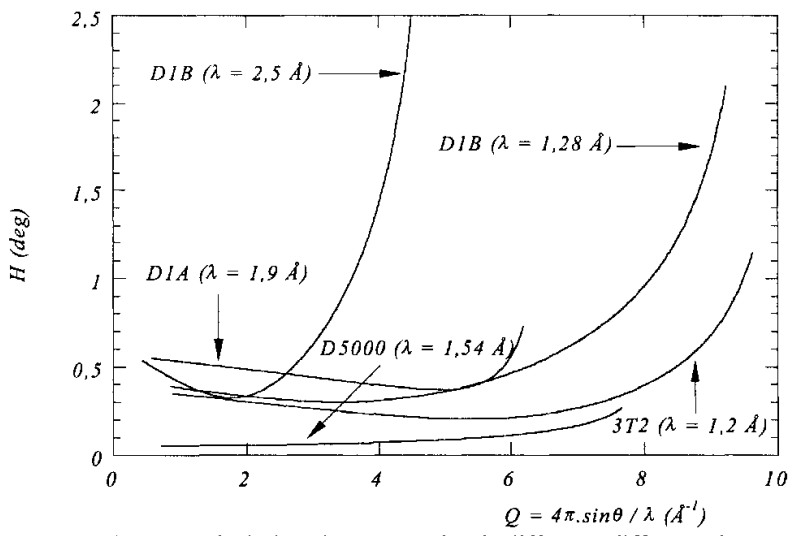

Figure 13: Comparaison des résolutions instrumentales de différents diffractomètres sur poudres.

\section{DIFFRACTION SUR MONOCRISTAL}

\subsection{Les diffractomètres}

La diffraction de neutrons sur monocristal est une technique très largement utilisée pour la détermination précise d'une structure cristalline. Il existe essentiellement deux catégories de diffractomètres adaptés à la diffraction sur monocristal : les 4-cercles et les diffractomètres en mode faisceau perpendiculaire. Les diffractomètres en mode faisceau perpendiculaire, permettant une inclinaison du détecteur hors du plan horizontal, autorisent en général l'installation d'équipements lourds pour des études in situ (cryostat, four, bobines supraconductrices, ...). Dans le cadre de ce cours, nous allons nous limiter au cas du diffractomètre 4-cercles dont le principe va être décrit brièvement.

La caractéristique essentielle d'un diffractomètre 4-cercles est de posséder trois berceaux de rotation d'Euler $\omega, \chi$ et $\varphi$. Ces trois angles permettent d'orienter le monocristal tel que le détecteur, souvent ponctuel, puisse se déplacer dans un plan horizontal. La Figure 14 présente le principe expérimental du diffractomètre 4-cercles 5C2 du LLB.

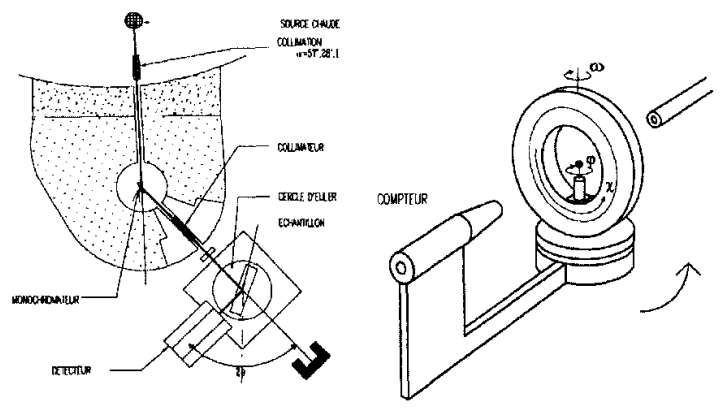

Figure 14: Schéma de principe du diffractomètre 4-cercles 5C2 (LLB).

A l'aide des trois angles $\omega, \chi$ et $\varphi$, le cristal est orienté de manière à amener un vecteur $\mathrm{du}$ réseau réciproque $\vec{Q}=\vec{k}_{\text {sor }}-\vec{k}_{\text {inc }}$ dans le plan horizontal contenant le détecteur (dont la position est repérée par l'angle 20 ). Conformément à ce qui a été développé dans le paragraphe 2.2 , en diffraction, la condition $\left\|\vec{k}_{\text {inc }}\right\|=\left\|\vec{k}_{\text {sor }}\right\|=\frac{2 \pi}{\lambda}$ est respectée. 
La Figure 15 résume les différentes étapes nécessaires à l'orientation convenable du vecteur de diffusion $\vec{Q}$ :

- (1) $\rightarrow$ (2) : la rotation du cristal d'un angle $\varphi$ permet d'amener le vecteur de diffusion $\vec{Q}$ dans un plan tangent à la sphère d'Ewald ;

- (2) $\rightarrow$ (3) : la rotation du cristal d'un angle $\chi$ permet d'amener le vecteur de diffusion $\vec{Q}$ dans le plan équatorial tout en restant dans le plan tangent à la sphère d'Ewald ;

- (3) $\rightarrow$ (4) : la rotation du cristal d'un angle $\omega$ permet d'amener le vecteur de diffusion $\vec{Q}$ en position de diffraction.

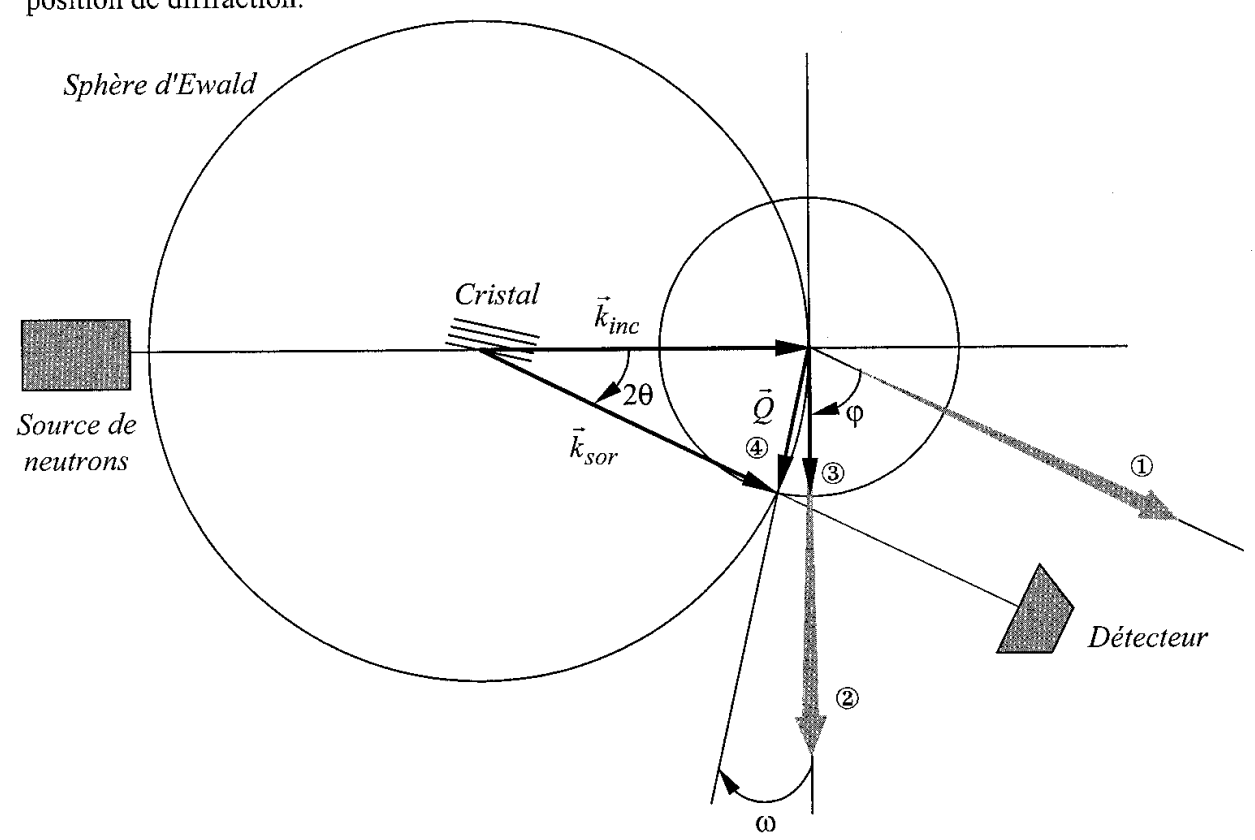

Figure 15: Etapes nécessaires à l'orientation du vecteur de diffusion $\vec{Q}$.

En résumé, compte tenu de sa géométrie, un diffractomètre 4-cercles permet de collecter individuellement l'intensité de chaque nœud du réseau réciproque.

\subsection{A quelles informations structurales a-t-on accès ?}

L'objectif d'une expérience de diffraction de neutrons sur monocristal est d'obtenir l'amplitude du facteur de structure $F_{h k l}$ pour une série de réflexions de Bragg $(h k l)$ de vecteur de diffusion $\vec{Q}$. Le module du facteur de structure $\left|F_{h k l}\right|$ est lié à l'intensité intégrée diffractée $I_{h k l}$ lorsque le cristal est amené en position de diffraction.

Dans le cas de la diffraction des neutrons l'intensité $I_{h k l}$ est égale à :

$$
I_{h k l}=\frac{\lambda^{3} \cdot V}{V_{c}^{2} \sigma} \cdot L \cdot A . T . E .\left|F_{h k l}\right|^{2}
$$

où $\lambda$ est la longueur d'onde, $V$ le volume du cristal, $V_{c}$ le volume de la maille élémentaire, $\sigma$ la vitesse angulaire de balayage, $L$ le facteur de Lorentz, $A$ le coefficient d'absorption, $T$ le facteur de 
correction de diffusion diffuse et $E$ le coefficient de correction d'extinction et de diffraction multiple.

Afin d'obtenir le module du facteur de structure $\left|F_{h k l}\right|$, il est nécessaire de mesurer aussi précisément que possible l'intensité diffractée $I_{h k l}$ et de la corriger des différentes erreurs (absorption, extinction, ...). Une expérience idéale est une expérience pour laquelle aucune correction n'est nécessaire (exceptée la correction de Lorentz qui est purement géométrique).

La collecte des intensités diffractées permet donc de déterminer la structure cristallographique du composé étudié. Plusieurs paramètres cristallographiques sont alors accessibles :

- les positions moyennes des atomes dans la structure permettant d'obtenir les distances interatomiques, les angles de liaison, les arrangements moléculaires...;

- les paramètres de déplacements atomiques donnant une information sur les vibrations thermiques moyennes et/ou le désordre atomique local;

- la distribution et l'arrangement des moments magnétiques dans le cas de structures magnétiques.

\subsection{Stratégie de collecte et de traitement des données}

\subsubsection{Collecte des données de diffraction}

En général, une expérience de diffraction de neutrons sur monocristal se déroule en quatre étapes : le choix du cristal, l'orientation du cristal, la stratégie de mesure et la collecte des données.

\section{- Choix du cristal}

Avant toute expérience, il est nécessaire de vérifier la qualité du monocristal en s'assurant qu'il s'agit bien d'un cristal unique, qui ne présente ni macles, ni cassures. Compte tenu de la faible section efficace de diffusion des neutrons par la matière, les échantillons étudiés sont en général de grande taille, i.e. au moins $1 \mathrm{~mm}^{3}$ (sauf dans le cas où l'échantillon contient un élément très absorbant).

\section{- Orientation du cristal}

Avant de démarrer une collecte de données de diffraction, il est nécessaire de repérer les axes du réseau réciproque par rapport au système d'axes du diffractomètre. Pour cela, on détermine une matrice $3 \times 3$ appelée matrice d'orientation. A partir de l'exploration automatique du réseau réciproque, on mesure plusieurs réflexions de Bragg dont les positions angulaires permettent de déduire la matrice d'orientation et les paramètres de maille.

\section{- Stratégie de mesure}

L'étape la plus importante consiste à adapter la stratégie de mesure à l'objectif de l'expérience en relation avec la fonction de résolution instrumentale du diffractomètre (cf. §3.6). Il s'avère donc nécessaire de connaître la projection, suivant la direction de balayage, de la fonction de résolution afin de déterminer la largeur du domaine angulaire balayé. D'autre part, il est nécessaire de déterminer le mode de balayage qui va déterminer le passage du nœud du réseau réciproque sur la sphère d'Ewald. On distingue deux modes de balayage : le " $\omega$ scan" et le " $\omega-2 \theta$ scan". Le premier mode consiste à faire toumer le cristal de $\omega-\Delta \omega$ à $\omega+\Delta \omega$ en laissant le détecteur fixe à la position $2 \theta$; dans le second mode, les rotations du cristal et du détecteur sont couplées telles que $\Delta 2 \theta=2 \Delta \omega$. Le choix du mode de scan dépend de la forme du noud réciproque.

\section{- Collecte des données de diffraction}

Pour la collecte des données de diffraction, il est nécessaire de définir la zone de l'espace réciproque à mesurer. Selon la symétrie cristalline de l'échantillon, certaines réflexions peuvent être équivalentes et il n'est alors pas nécessaire de les mesurer individuellement (sauf si l'on souhaite 
vérifier a posteriori la symétrie cristalline). De plus, au cours de la collecte, il est courant de mesurer régulièrement des réflexions standards (ou réflexions de référence) qui permettent de corriger d'éventuelles dérives instrumentales ou de détecter une dégradation progressive du cristal. Enfin, il existe deux modes de comptage : comptage au temps (on fixe le temps de comptage) ou comptage au moniteur (on fixe le nombre de neutrons incidents traversant le moniteur).

\subsubsection{Réduction et traitement des données}

Une fois le scan effectué sur un domaine angulaire, il est nécessaire d'extraire l'intensité intégrée du nœud du réseau réciproque. On utilise en général une méthode d'intégration point par point qui nécessite toutefois une estimation précise du bruit de fond de part et d'autre de la raie (Fig. 16).

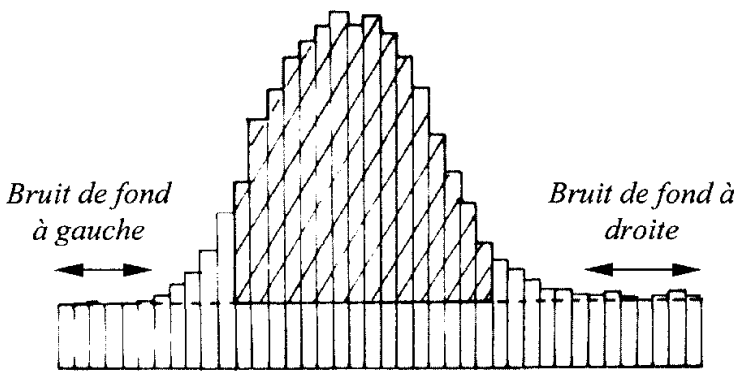

Figure 16: Estimation du bruit de fond et intégration de l'intensité d'un nœud du réseau réciproque.

De cette intégration, on tire ainsi l'intensité $I_{h k l}$ qui permettra de remonter au module du facteur de structure $\left|F_{h k l}\right|$. Cette opération nécessite que l'intensité soit préalablement corrigée des différentes erreurs citées précédemment (absorption, ...). Une fois les intensités corrigées, les réflexions équivalentes par symétrie sont moyennées. Il est alors possible d'extraire les modules des facteurs de structure $\left|F_{h k l}\right|$ qui constituent les données de base de la détermination structurale.

La détermination d'une structure à partir de données de diffraction collectées sur un 4-cercles est une procédure très efficace dans la plupart des cas. En général, les paramètres de maille sont obtenus par moindres carrés (lors de la détermination de la matrice d'orientation) et le groupe d'espace est déduit des extinctions systématiques. Ces informations peuvent être éventuellement obtenues à partir d'une étude préalable par diffraction des rayons $\mathrm{X}$ (chambre de Weissenberg, chambre de précession, Laue...).

Le modèle structural de départ est obtenu en appliquant les techniques de Patterson, les méthodes directes, la méthode de l'atome lourd... Les paramètres structuraux initiaux sont alors affinés et les atomes supplémentaires sont révélés par des techniques de Fourier différence.

\subsection{Comparaison de différents instruments}

Les diffractomètres 4-cercles se distinguent par leur longueur d'onde de travail déterminant le degré de précision de la détermination structurale :

- Les courtes longueurs d'onde (neutrons chauds) sont dédiées à la détermination précise des positions atomiques, des paramètres de déplacements atomiques et des moments magnétiques dans des structures dont le volume de la maille est inférieur à $1000 \AA^{3}$. Grâce à la longueur d'onde très courte, il est possible de déterminer des déplacements atomiques très faibles (jusqu'à $0,001 \AA$ ) et de calculer des cartes de densité nucléaire très précises. Les diffractomètres D9 de l'TLL et 5C2 du LLB répondent à ces caractéristiques. Le 4-cercles D10 
de l'ILL est très bien adapté à l'étude des structures modulées et sa configuration trois-axes permet également des études de diffusion inélastiques.

- Les longueurs d'onde moyennes (neutrons thermiques) sont réservées à l'étude de grandes structures dont le volume de maille est compris entre 1000 et $10000 \AA^{3}$. Les diffractomètres D19 à l'ILL et 6T2 au LLB permettent ce genre d'études.

- Enfin, les grandes longueurs d'onde (neutrons froids) sont dédiées à l'étude de très grandes structures telles que les macromolécules biologiques par exemple. Le diffractomètre D11 de l'ILL permet, grâce à son sélecteur de vitesse, de travailler avec des longueurs d'onde comprises entre 4,5 et $40 \AA$.

Il apparaît clairement que le choix du diffractomètre est conditionné par la problématique de l'étude. Une fois l'instrument défini, la longueur d'onde de travail est ensuite sélectionnée de façon à obtenir le meilleur compromis entre le flux et la résolution.

\subsection{Quelques exemples d'application}

\subsubsection{Résolution d'une structure nucléaire - Cartes de densités nucléaires}

Les BIMEVOX sont des matériaux appartenant à la famille des conducteurs à ions $\mathrm{O}^{2-}$ et sont formulés $\mathrm{Bi}_{4}\left(\mathrm{~V}_{1-\mathrm{x}} \mathrm{Me}_{\mathrm{x}}\right)_{2} \mathrm{O}_{11-\delta}$, où Me est en général un métal de transition. Ils dérivent du composé $\mathrm{Bi}_{4} \mathrm{~V}_{2} \mathrm{O}_{1 /}$ dont la structure s'apparente à celle de la phase d'Aurivillius $\mathrm{Bi}_{2} \mathrm{MoO}_{6}$ et cristallisent sous forme d'alternance de feuillets rigides $\left(\mathrm{Bi}_{2} \mathrm{O}_{2}\right)^{2+}$ entre lesquels s'intercalent des couches d'octaèdres de type perovskite $\left[(\mathrm{V}, \mathrm{Me})(\mathrm{O}, \square)_{4}\right]^{2-}$. La substitution partielle du vanadium par un métal Me a pour effet de stabiliser, à température ambiante, la phase $\gamma^{\prime}$ isomorphe de la phase $\gamma$ haute température du composé $\mathrm{Bi}_{4} \mathrm{~V}_{2} \mathrm{O}_{11}$.

Une attention particulière a été portée sur le composé $\mathrm{Bi}_{4}\left(\mathrm{~V}_{0,85} \mathrm{Co}_{0,15}\right)_{2} \mathrm{O}_{11-\delta}$, appelé BICOVOX.15, qui présente une transition de phase vers $780 \mathrm{~K}$ entre une phase désordonnée haute température $\gamma$ et une phase ordonnée de plus basse température $\gamma$. La structure cristallographique moyenne de la phase $\gamma^{\prime}$ a été étudiée par diffraction de neutrons sur monocristal [17]. Les études précédentes, effectuées à partir de données de diffraction de rayons $\mathrm{X}$, s'étaient heurtées au problème délicat de localisation de l'oxygène dans cette structure partiellement désordonnée. Compte tenu de la longueur de diffusion cohérente favorable de l'oxygène, la diffraction des neutrons permet de résoudre ce problème.

La collecte des intensités a été effectuée sur un monocristal de $4 \mathrm{~mm}^{3}$ environ, à température ambiante, sur le diffractomètre 4-cercles DN4 à Silöe (CEN - Grenoble) avec une longueur d'onde de $1,18 \AA .591$ réflexions ont été collectées en mode " $\omega s c a n "\left(2^{\circ} \leq \theta \leq 45^{\circ}\right)$ et 473 en mode $" \omega-2 \theta \operatorname{scan}^{\prime}\left(40^{\circ} \leq \theta \leq 60^{\circ}\right)$. Ces 1064 réflexions ont ensuite été moyennées dans le groupe d'espace quadratique $14 / \mathrm{mmm}$ pour donner 137 réflexions indépendantes servant de données de base pour la détermination structurale.

Cette étude a permis :

- de confirmer l'absence de lacunes d'oxygène dans les feuillets $\mathrm{Bi}_{2} \mathrm{O}_{2}$;

- de mettre en évidence des densités nucléaires allongées, caractéristiques de la diffusion de l'oxygène, formant ainsi des chemins de diffusion circulaires joignant les sites des atomes d'oxygène apicaux $O(2)$ et équatoriaux $O(3)$ (Fig. 17);

- de révéler une distribution de lacunes plus importante sur les sites équatoriaux $O(3)$ que sur les sites apicaux $\mathrm{O}(2)$;

- de préciser la formule chimique du composé et la stæchiométrie en oxygène, i.e. $\mathrm{Bi}_{4}\left(\mathrm{~V}_{0,835} \mathrm{Co}_{0,125} \mathrm{Bi}_{0.04}\right)_{2} \mathrm{O}_{10,545}$. 


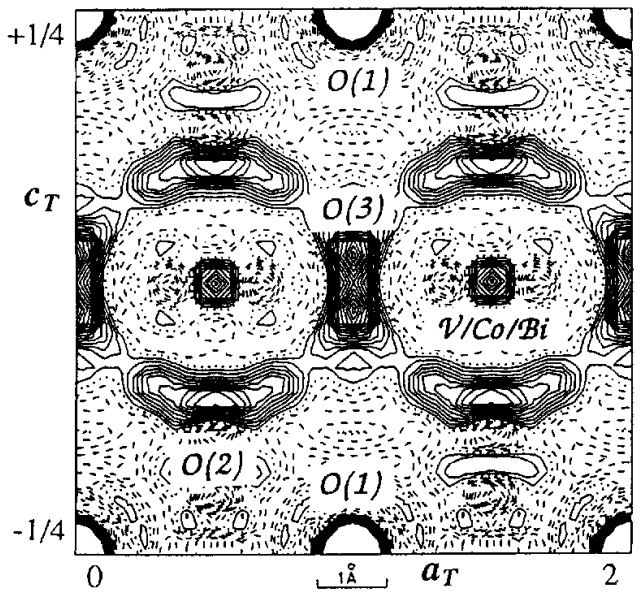

Figure 17: Carte de densités nucléaires mettant en évidence des chemins de diffusion circulaires joignant les différents sites cristallographiques des atomes d'oxygène [17].

Les mouvements localisés de l'oxygène, mis en évidence par diffusion quasiélastique de neutrons [18], peuvent être expliqués par un échange des atomes d'oxygène des sites $O(2)$ et $O(3)$. Le modèle de diffusion le plus probable consiste en une rotation uniaxiale des anions $\mathrm{O}^{2-}$ sur un cercle, l'axe de rotation étant parallèle à l'un des axes cristallographiques $a_{T}$ ou $b_{T}$. Les cercles de diffusion étant connectés par les sites $\mathrm{O}(2)$ et $\mathrm{O}(3)$, les anions peuvent sauter d'un cercle à l'autre, donnant ainsi naissance à une diffusion à longue distance.

\subsubsection{Résolution d'une structure magnétique}

Comme cela a été rappelé au paragraphe 2 , le neutron possède un spin $1 / 2$ susceptible d'interagir avec le moment magnétique porté par un atome. Dans bon nombre de composés, il apparaît, endessous d'une certaine température, un ordre des moments magnétiques (ordre ferromagnétique ou antiferromagnétique par exemple...). En général, cette structure, définie par une maille magnétique qui peut être multiple de la maille nucléaire, est mise en évidence par diffraction de neutrons, les réflexions magnétiques venant se superposer aux réflexions nucléaires dans le cas d'un ordre ferromagnétique ou apparaissant entre les réflexions nucléaires dans le cas d'un ordre antiferromagnétique.

A titre d'illustration, les structures nucléaire et magnétique de l'alliage $\mathrm{Nd}_{2} \mathrm{Fe}_{14} \mathrm{~B}$ ont été étudiées précisément par diffraction de neutrons sur monocristal. Ce composé est le prototype d'une série d'aimants permanents présentant une très forte aimantation associée à la forte densité d'atomes de fer et une très forte anisotropie magnétocristalline liée à la présence de néodyme dans la structure. Cet alliage présente une transition de réorientation de spin à $140 \mathrm{~K}$ entre deux phases ferromagnétiques de symétrie quadratique à température ambiante (groupe d'espace $P 4_{2} / \mathrm{mmm}$ ) et de symétrie monoclinique à basse température (groupe d'espace $\mathrm{Cm}$ ). Dans la phase quadratique, l'axe de facile aimantation est parallèle à l'axe quadratique $\vec{c}$ [19].

Des données de diffraction de neutrons ont été collectées à $20 \mathrm{~K}$ sur le diffractomètre 4-cercles D10 à l'ILL [20]. Les réflexions de Bragg ont été mesurées sur la demi-sphère du réseau réciproque jusqu'à $\frac{\sin \theta}{\lambda} \approx 0,61 \AA^{-1}$. Le choix d'une longueur d'onde de $1,26 \AA$ a permis d'obtenir un bon compromis entre la résolution instrumentale, le flux et la contamination par les harmoniques. Le 
bore étant un élément très absorbant, le cristal a été rendu sphérique afin de faciliter les corrections d'absorption. La Figure 18 présente un schéma de la structure ferromagnétique de l'alliage $\mathrm{Nd}_{2} \mathrm{Fe}_{14} \mathrm{~B}$ à $20 \mathrm{~K}$.

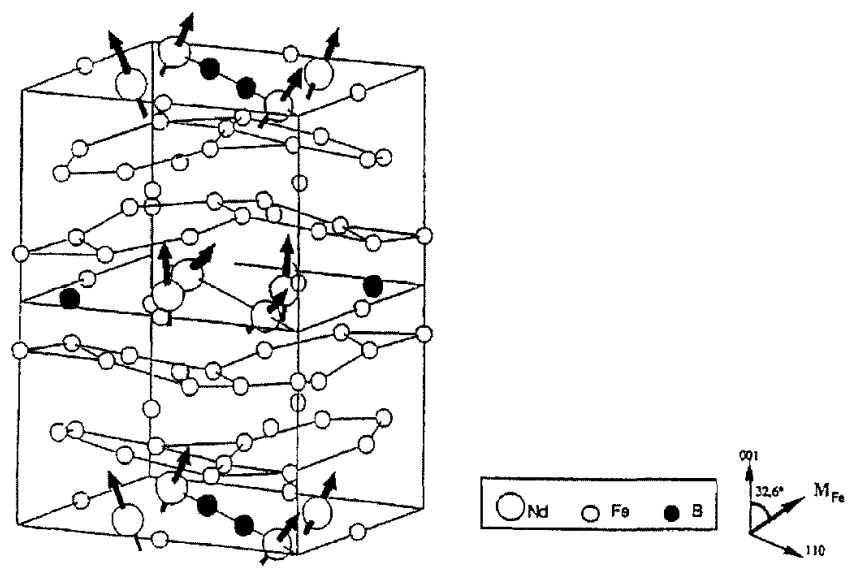

Figure 18: Structure ferromagnétique de l'alliage $\mathrm{Nd}_{2} \mathrm{Fe}_{14} \mathrm{~B}$ à $20 \mathrm{~K}$ [20].

Dans la phase ferromagnétique basse température de symétrie monoclinique, l'axe de facile aimantation n'est pas colinéaire avec le pseudo-axe d'ordre 4 mais il est basculé de $32^{\circ}$ environ par rapport à la direction de l'axe $\vec{c}$ quadratique. Sur la Figure 18, les moments magnétiques portés par les atomes de fer sont tous pris parallèles entre eux et parallèles à l'aimantation résultante des atomes de néodyme.

\subsubsection{Etude d'un cristal par la méthode de Laue}

L'alliage $\mathrm{La}_{2} \mathrm{Co}_{1,7}$ est un composé antiferromagnétique de symétrie hexagonale présentant une transition de phase paramagnétique $\rightarrow$ antiferromagnétique à $146 \mathrm{~K}$ [21]. Wilkinson et al. ont effectué une étude par diffraction de neutrons sur monocristal ayant pour objectif de confirmer l'existence d'une structure nucléaire incommensurable liée à l'arrangement des atomes de cobalt le long de l'axe hexagonal [12].

Comme nous l'avons vu dans les deux exemples précédents, les structures nucléaires et magnétiques sont souvent étudiées par diffraction de neutrons monochromatiques sur monocristal. Toutefois, même sur un réacteur à haut flux, la collecte d'un jeu complet de données de diffraction, pouvant inclure plusieurs milliers de réflexions satellites pour une structure incommensurable, peut parfois prendre plusieurs semaines. Une alternative possible est l'utilisation de la technique de Laue en faisceau blanc, consistant à amener sur le monocristal des neutrons polychromatiques. L'utilisation combinée de cette technique et d'un détecteur bidimensionnel permet de réduire le temps d'acquisition à quelques heures. Cette technique a l'avantage supplémentaire d'explorer tout l'espace réciproque et permet ainsi de mesurer des réflexions additionnelles qui ne seraient pas forcément prises en compte dans une collecte classique sur un diffractomètre 4-cercles.

L'alliage $\mathrm{La}_{2} \mathrm{Co}_{1,7}$ a été étudié sur le diffractomètre quasi-Laue LADI de l'ILL (guide de neutrons froids S42) qui ne sélectionne en fait qu'un domaine limité de longueurs d'onde afin de réduire le nombre de réflexions simultanément actives sur le détecteur (d'où la dénomination de quasi-Laue). L'utilisation de l'image plate décrite dans le paragraphe 3.5.4. a permis de réduire les temps d'acquisition à une trentaine de minutes. Afin d'augmenter la résolution sur les réflexions satellites, un filtre formé de multicouches NiTi a été utilisé de façon à limiter le domaine de longueur d'onde à 
$2,5 \AA<\lambda<3,5 \AA$. La Figure 19 présente un diagramme de Laue de l'alliage $\mathrm{La}_{2} \mathrm{Co}_{1,7}$ à $15 \mathrm{~K}:$ on discerne nettement les réflexions fondamentales intenses des groupes hexagonaux de réflexions satellites plus faibles.

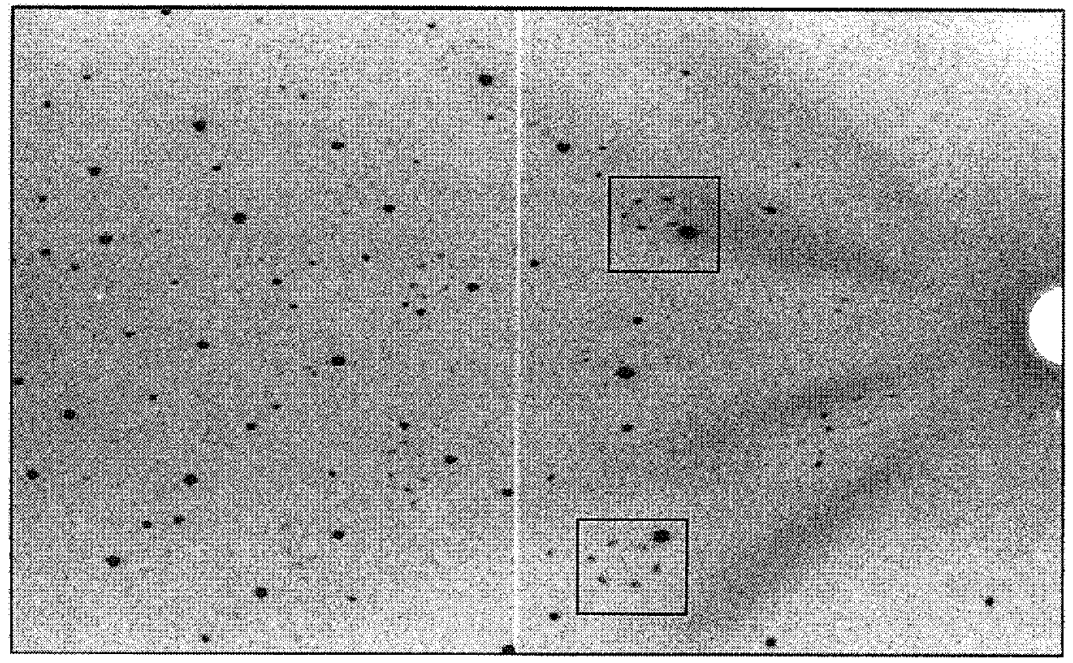

Figure 19: Diagramme de Laue enregistré sur l'alliage $\mathrm{La}_{2} \mathrm{Co}_{1,7}$ à $15 \mathrm{~K}$.

La modulation de la phase incommensurable est décrite par 6 vecteurs de propagation, chaque vecteur donnant naissance à deux réflexions satellites. Ceci explique les petits îlots hexagonaux de réflexions satellites observés à proximité des réflexions fondamentales (îlots encadrés sur la Figure 19). L'utilisation de programmes spécifiques a permis ensuite d'indexer et d'intégrer les intensités sur les diagrammes de Laue afin de résoudre la structure et d'affiner les composantes des vecteurs de propagation.

\subsubsection{Diffraction de fibres : exemple de la cellulose}

La technique de diffraction de neutrons est certes très utilisée dans le domaine des matériaux, mais elle intéresse également la biologie structurale. En effet, elle représente une technique très riche en particulier pour l'étude des polymères fibreux ou les biopolymères [22]. Alors que l'hydrogène diffuse très peu en diffraction des rayons $X$, il est un très fort diffuseur aux neutrons et le pouvoir diffusant de son isotope le deutérium est encore plus élevé. La diffraction des neutrons est par conséquent une technique efficace pour la localisation des molécules d'eau dans des polymères biologiques hydratés. Dans de nombreuses études, le remplacement de $\mathrm{H}_{2} \mathrm{O}$ par $\mathrm{D}_{2} \mathrm{O}$ dans les fibres est mis à profit afin d'augmenter le pouvoir diffusant des molécules d'eau. De plus, l'autre avantage important des neutrons est que la longueur de diffusion cohérente ne dépend pas de l'angle de diffusion contrairement au facteur de forme des rayons $\mathrm{X}$. Cette caractéristique permet d'obtenir des diagrammes de diffraction de meilleure qualité aux grands angles de diffraction.

Afin d'illustrer l'utilisation de la diffraction sur des fibres, nous allons décrire brièvement l'expérience effectuée par Nishiyama et al. sur de la cellulose [23]. Cette étude a été menée sur le diffractomètre 4-cercles D19 à l'ILL. Les diagrammes de diffraction ont été collectés à l'aide d'un détecteur à gaz bidimensionnel sur des celluloses hydratées et deutérées. Pour une cellulose donnée, un contraste évident apparaît entre l'échantillon hydraté noté $\mathrm{OH}$ et l'échantillon deutéré noté OD (Fig. 20). La variation d'amplitudes mesurées permet ensuite de construire des cartes de différence de Fourier sur lesquelles sont localisées les molécules d'eau. 


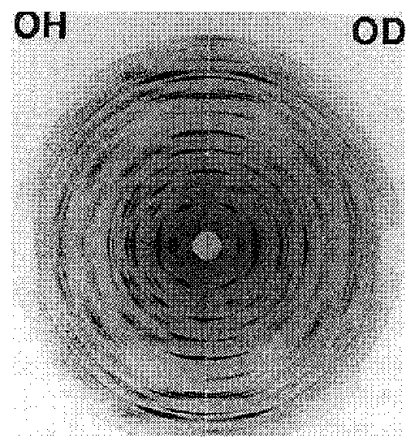

Figure 20: Diagrammes de diffraction de fibres enregistrés sur des celluloses hydratée (OH) et deutérée (OD) [23].

\subsubsection{Exemple d'un diffractomètre sur source à spallation}

Le diffractomètre 4-cercles TriCS à SINQ (source à spallation Suisse) est dédié à la résolution de structures nucléaires et magnétiques de composés ayant des paramètres de maille inférieurs à $20 \AA$ [24]. Cet instrument possède deux monochromateurs permettant de travailler soit en mode haute résolution pour les structures nucléaires (monocristal de germanium (311)), soit en mode haut flux pour les structures magnétiques (monocristal de graphite (002)). La Figure 21a présente le schéma de principe du diffractomètre.

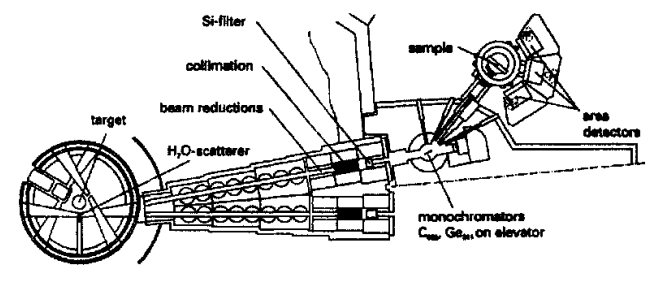

a)

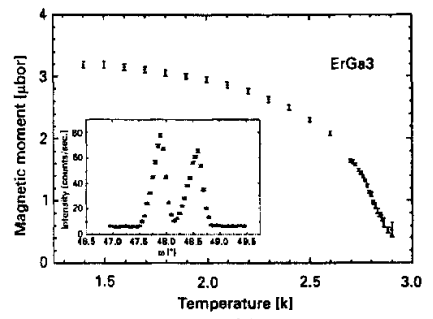

b)

Figure 21: (a) Schéma de principe du diffractomètre 4-cercles TriCS (SINQ).

(b) Evolution thermique du moment magnétique porté par les atomes d'erbium [24].

L'une des premières expériences sur TriCS a effectuée sur un monocristal de $\mathrm{ErGa}_{3}$. Cet alliage cristallise dans une structure cubique du type $\mathrm{AuCu}_{3}$, dans laquelle les atomes d'erbium occupent les sommets du cube et les atomes de gallium les milieux de faces. En-dessous de $2,8 \mathrm{~K}$ environ apparaît un ordre des moments magnétiques portés par les atomes de terre rare [25]. Les mesures effectuées sur monocristal par diffraction de neutrons (Fig. 21b) attestent que l'aimantation s'annule au-dessus d'une température de $2,8 \mathrm{~K}$ environ [24].

\section{DIFFRACTION SUR POUDRES}

\subsection{Principe}

La dénomination de poudre est communément utilisée, mais en réalité, il est plus rigoureux d'employer le terme d'échantillon polycristallin. Un échantillon polycristallin idéal est constitué d'un grand nombre de petits cristallites orientés aléatoirement les uns par rapport aux autres, le nombre de cristallites en position de diffraction étant toujours identique.

Compte tenu de cette distribution aléatoire, il existe toujours plusieurs cristallites qui ont une orientation respectant la condition de diffraction. Il en résulte que les faisceaux diffractés se placent à la surface d'un cône, appelé cône de Debye (Fig. 22). 


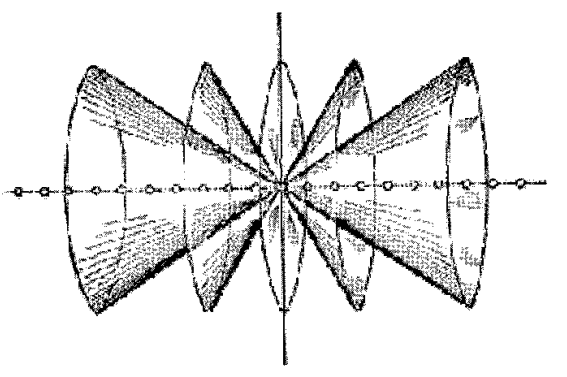

Figure 22: Représentation des cônes de Debye.

Contrairement aux diffractomètres 4-cercles qui permettent de mesurer individuellement chaque nœud du réseau réciproque, les diffractomètres sur poudres ne donnent qu'une projection à une dimension de l'espace réciproque tridimensionnel. Par rapport à une collecte sur monocristal, il y a donc une perte d'information liée notamment à la superposition des réflexions équivalentes. Classiquement, les données de diffraction sont obtenues sous la forme d'un diagramme donnant le nombre de neutrons comptés pour chaque angle $2 \theta$.

Les instruments utilisés en diffraction de neutrons sont des diffractomètres 2-axes configurés en géométrie Debye-Scherrer en référence à la géométrie utilisée dans les chambres du même nom en diffraction des rayons $\mathrm{X}$. Ces diffractomètres sont en général équipés d'un multidétecteur fixe ou mobile permettant d'intercepter une partie des cônes de Debye. A titre d'illustration, la Figure 23 présente le schéma de principe du diffractomètre à haut flux DIB de l'ILL. Cet instrument est équipé d'un multidétecteur PSD fixe formé de 400 cellules couvrant un domaine angulaire de $80^{\circ}$ en $2 \theta$. Sa résolution instrumentale étant meilleure aux petits angles (cf. Fig. 13), cet instrument était dédié à l'origine à l'étude de structures magnétiques. Il s'avère toutefois que le haut flux de neutrons disponible fait de cet instrument un outil très performant pour les études in situ et en temps réel. Le temps d'acquisition moyen d'un diagramme est de l'ordre de 5 minutes à $\lambda=2,5 \AA$ et d'une heure à $\lambda=1,28 \AA$.

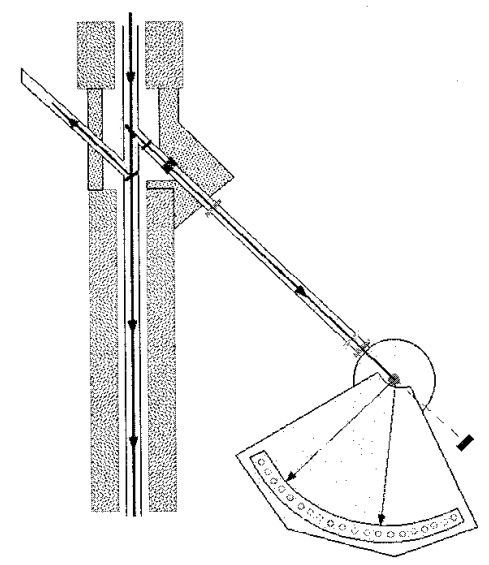

Figure 23: Schéma de principe du diffractomètre à haut flux D1B (ILL).

\subsection{Traitement des données de diffraction - Méthodes d'ajustement de profil}

Comparativement à la diffraction sur monocristal, la principale difficulté de la diffraction sur poudres réside dans la réduction à une dimension des trois dimensions de l'espace réciproque. Il en 
résulte un grand recouvrement angulaire des réflexions de Bragg lié en particulier à l'élargissement de la résolution instrumentale. Il est donc très délicat de déterminer précisément les contributions respectives de chaque réflexion sur un domaine angulaire donné. La Figure 24 présente deux diagrammes, d'un même échantillon, simulés respectivement avec des largeurs instrumentales de 0,1 et $0,5^{\circ}$ en $2 \theta$. La différence observée illustre la nécessité de travailler avec une résolution élevée en diffraction sur poudres. Cette résolution doit être d'autant plus grande que la symétrie du composé est faible. Toutefois, l'utilisation de méthodes d'ajustement de profil performantes permet d'extraire les intensités $I_{h k l}$ nécessaires à la résolution structurale.

Les différentes étapes de la détermination $a b$ initio d'une structure cristalline vont être décrites brièvement dans les paragraphes suivants [26].

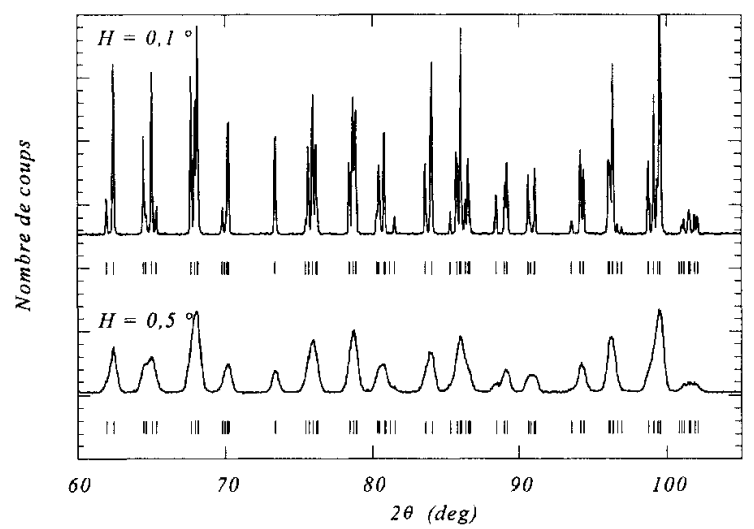

Figure 24: Effet de la résolution instrumentale sur la séparation des réflexions.

\subsubsection{Ajustement individuel des profils de raies}

Cette méthode consiste à ajuster une fonction analytique sur un profil expérimental sans aucune référence à la maille ou à la structure cristallographique du composé. La seule contrainte est liée au choix du profil analytique, dont l'intensité, la position angulaire et la largeur à mi-hauteur sont des paramètres ajustables par une méthode de minimisation. Le bruit de fond est en général pris en compte à l'aide d'un polynôme de degré 0,1 ou 2 . La Figure 25 présente un exemple d'ajustement individuel des profils de deux réflexions de Bragg.

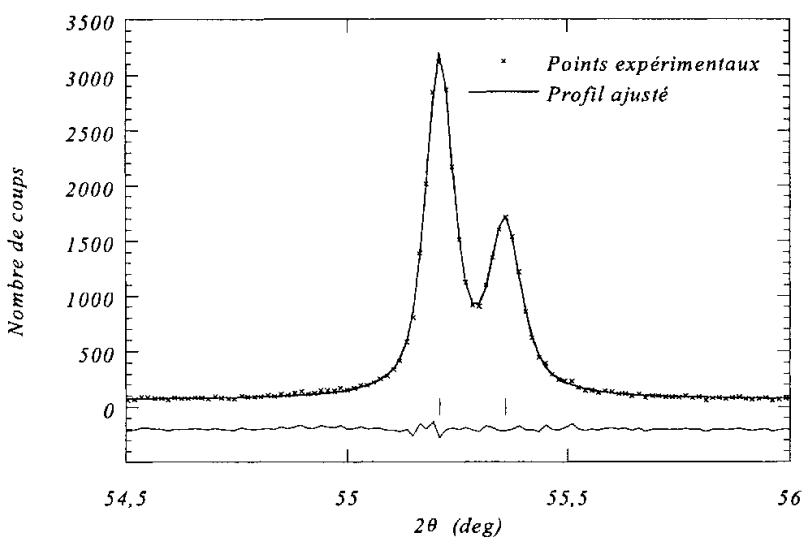

Figure 25: Ajustement individuel des profils de raies de diffraction. 
A partir de cette technique, il est possible d'obtenir précisément les positions des réflexions qui, par le biais de méthodes d'indexation automatique, permettent de déterminer les paramètres de maille et l'indexation de chacune des réflexions de Bragg. L'observation des extinctions systématiques des réflexions conduit alors à la proposition d'un ou plusieurs groupes d'espace.

\subsubsection{Ajustement de profil total par contrainte de maille}

Dans cette méthode, le profil total, c'est-à-dire l'ensemble du diagramme, est analysé sans référence à un modèle structural, mais nécessite la connaissance approximative des paramètres de maille, des paramètres de profil $U, V$ et $W$ (cf. \$3.6.2) et du groupe d'espace. Dans cette approche, les positions des raies sont contraintes par les paramètres de maille et les intensités sont des variables libres.

Le nombre de coups observé à chaque pas angulaire $i$ du diagramme est modélisé par :

$$
y_{i}^{\text {calc }}=y_{i}^{b}+\sum_{k=k_{I}}^{k_{2}} I_{k} \cdot \Omega_{i k}
$$

où

$y_{i}^{b}$ représente l'intensité du bruit de fond au pas $i$

$\Omega_{i k}=\Omega\left(2 \theta_{i}-2 \theta_{k}\right)$ est la fonction décrivant le profil expérimental

$I_{k}$ est l'intensité intégrée de la raie $k$ contribuant au pas angulaire $i$

Cette technique est donc utilisée pour affiner les paramètres de maille obtenus par des méthodes d'indexation automatique, pour tester les différents groupes d'espace possibles et pour extraire les intensités intégrées $I_{h k l}$ proportionnelles à $\left|F_{h k l}\right|^{2}$.

\subsubsection{Détermination d'un modèle structural}

Une fois la symétrie du composé connue, il est parfois possible d'identifier la structure par comparaison avec d'autres composés de la littérature. Si tel est le cas, on peut alors utiliser directement une méthode d'affinement de structure par la méthode de Rietveld (cf. \$5.2.4). Toutefois, dans de nombreux cas, aucun isotype ne peut être trouvé et il est alors nécessaire d'employer les mêmes méthodes que celles utilisées pour la résolution structurale à partir d'une collecte sur un diffractomètre 4-cercles (méthodes directes, méthode de Patterson, méthode de l'atome lourd...).

\subsubsection{Affinement de structure par ajustement de profil total - Introduction à la méthode de Rietveld}

Cette méthode, initiée par Rietveld en 1969, est la procédure d'affinement de structure la plus courante lorsque la structure d'un échantillon est approximativement connue [27-30]. La procédure d'affinement minimise la fonction $M$ par les méthodes de moindres carrés ou de maximum de vraisemblance :

$$
M=\sum_{i} w_{i}\left(y_{i}^{o b s}-y_{i}^{c a l c}\right)^{2}
$$

où $w_{i}=\frac{1}{\sigma_{i}^{2}}$ est la pondération affectée à l'observable $y_{i}^{o b s}$ (nombre de coups observés au pas i) dont $\sigma_{i}$ est l'écart type et $y_{i}^{\text {calc }}$ le nombre de coups calculés au pas $i, y_{i}^{c a l c}$ étant défini comme :

$$
y_{i}^{c a l c}=y_{i}^{b}+\sum_{\ell} S_{\ell} \sum_{k} m_{\ell k} \cdot L_{\ell k} \cdot O_{\ell k}\left|F_{\ell k}\right|^{2} \cdot \Omega_{i \ell k} \cdot T
$$

où $\quad \ell$ est l'indice de sommation sur les différentes phases

$k$ est l'indice de sommation sur toutes les réflexions voisines contribuant au pas $i$

$S_{\ell}$ est le facteur d'échelle entre les valeurs observées et les valeurs calculées 
$m_{\ell k}$ est la multiplicité de la $k^{\text {ème }}$ réflexion

$L_{\ell k}$ est le facteur de Lorentz

$O_{\ell k}$ est le facteur décrivant les effets d'orientation préférentielle

$\mathrm{F}_{\ell \mathrm{k}}$ est le facteur de structure

$T$ est le facteur d'absorption

La minimisation de cette fonction permet d'obtenir les positions des atomes dans la maille, ainsi que les paramètres de déplacements atomiques (vibrations thermiques et désordre local) qui leurs sont associés. Il est également possible d'affiner les taux d'occupation des atomes sur leurs sites cristallographiques.

\subsubsection{Affinement en mode séquentiel}

Une méthode d'affinement séquentiel peut être utilisée lorsque l'on souhaite obtenir l'évolution de certains paramètres structuraux en fonction de la température par exemple. Le principe est de modéliser le premier diagramme d'une série de données de diffraction et d'ajuster successivement tous les diagrammes en utilisant comme paramètres de départ les paramètres affinés sur le diagramme précédent. On suppose, pour cela, que les modifications sont faibles d'un diagramme à l'autre.

\subsection{Comparaison de différents instruments}

Les diffractomètres 2-axes sont dédiés à l'étude des échantillons polycristallins, des liquides et des matériaux amorphes et sont installés en général sur des sources chaudes ou thermiques. Le tableau ci-dessous compare les caractéristiques de trois instruments D2B, D1B et D4 de l'ILL.

\begin{tabular}{|c|c|c|c|c|c|c|}
\hline & \multicolumn{2}{|c|}{ D2B } & \multicolumn{2}{c|}{ D1B } & \multicolumn{2}{c|}{ D4 } \\
\hline$\lambda(\AA)$ & 1,6 & 2,4 & 1,28 & 2,5 & 0,35 & 0,7 \\
\hline$Q_{\min }\left(\AA^{-1}\right)$ & 0,3 & 0,2 & 0,2 & 0,1 & 0,3 & 0,2 \\
\hline$Q_{\max }\left(\AA^{-1}\right)$ & 7,9 & 5,2 & 9,0 & 4,8 & 34 & 17 \\
\hline $\begin{array}{c}\text { Temps de } \\
\text { comptage }\end{array}$ & 1 heure & 2 heures & 1 heure & 5 minutes & 10 heures & 1 heure \\
\hline $\begin{array}{c}\text { Résolution } \\
\frac{\Delta d}{d} \times 10^{3}\end{array}$ & $\begin{array}{c}0,5 \text { (mode haute } \\
\text { résolution) } \\
1,5 \text { (mode haut flux) }\end{array}$ & \multicolumn{2}{|c|}{6} & & 20 \\
\hline Applications & $\begin{array}{c}\text { Résolutions structurales } \\
a b \text { initio }\end{array}$ & Etudes in situ et temps réel & $\begin{array}{c}\text { Etudes de liquides et de } \\
\text { matériaux amorphes }\end{array}$ \\
\hline
\end{tabular}

La très bonne résolution instrumentale et le haut flux font de $\mathrm{D} 2 \mathrm{~B}$ un instrument très performant pour la détermination $a b$ initio de structures cristallines dont le volume de la maille n'excède pas $1000 \AA^{3}$. Le diffractomètre D1B, qui a déjà été présenté (cf. Fig. 23), ne possède qu'une résolution moyenne, mais son haut flux est très bien adapté aux expériences in situ et temps réel (temps d'acquisition moyen d'un diagramme à 2,5 $\AA$ : 5 minutes). Enfin, le diffractomètre D4, placé sur une source chaude, est configuré pour travailler avec de courtes longueurs d'onde permettant l'acquisition de diagramme de diffraction sur un très grand domaine en $Q$. Il assure donc la caractérisation très précise de l'ordre atomique local dans les liquides ou les matériaux amorphes. 


\subsection{Quelques exemples d'application}

\subsubsection{Transitions de phase structurales - Etude par thermodiffractométrie des neutrons sur poudre}

Les composés $\mathrm{PbHfO}_{3}$ et $\mathrm{PbTiO}_{3}$ sont intéressants, d'une part pour leurs propriétés électriques intrinsèques, mais également parce que leur association conduit à la formation d'une solution solide $\mathrm{PbHf}_{1-\mathrm{x}} \mathrm{Ti}_{\mathrm{x}} \mathrm{O}_{3}$ (PHT), pour laquelle on obtient des propriétés diélectriques attractives. Ces nouveaux matériaux constituent une alternative aux $\mathrm{PZT}\left(\mathrm{PbZr}_{1-\mathrm{x}} \mathrm{Ti}_{\mathrm{x}} \mathrm{O}_{3}\right)$, proches structuralement et qui, à l'heure actuelle, ont de très nombreuses applications industrielles.

Le diagramme de phase binaire $\mathrm{PbHfO}_{3}-\mathrm{PbTiO}_{3}$ présente une grande variété de transitions de phase entre des états électriques divers (ferroélectrique, antiferroélectrique ou paraélectrique). Ces transitions mettent en jeu des déplacements cationiques et des déformations des octaèdres d'oxygène conduisant à des structures de type perovskite déformée.

L'étude structurale fine des transitions de phase dans ces composés a nécessité l'utilisation conjuguée de deux diffractomètres neutroniques de résolutions différentes. La haute résolution de l'instrument 3T2 du LLB (cf. Fig. 8) a permis l'acquisition de diagrammes de très bonne qualité entre chaque transition de phase. Les affinements structuraux, effectués par la méthode de Rietveld, ont permis de valider l'étude in situ des transitions de phase menée sur l'instrument de plus faible résolution D1B (ILL) avec $\lambda=2,5 \AA$ (cf. Fig. 23). Le haut flux disponible et le multidétecteur de 400 cellules de ce diffractomètre ont permis de suivre précisément les transitions et de déterminer, par traitement séquentiel des diagrammes, l'évolution des paramètres structuraux sur une gamme de température s'étalant en général de 300 à $800 \mathrm{~K}$. A titre d'illustration, la Figure 26 présente l'évolution thermique des diagrammes de diffraction enregistrés sur l'échantillon $\mathrm{PbHf}_{0,2} \mathrm{Ti}_{0,8} \mathrm{O}_{3}$. $\mathrm{Ce}$ composé présente une transition de phase vers $670 \mathrm{~K}$ entre une phase ferroélectrique de symétrie quadratique et une phase paraélectrique de symétrie cubique [31]. L'évolution des diagrammes montre clairement que les deux réflexions $(200)_{\mathrm{q}}$ et $(002)_{\mathrm{q}}$ de la phase quadratique converge progressivement pour donner un singulet (réflexion $(200)_{c}$ ) dans la phase cubique haute température.

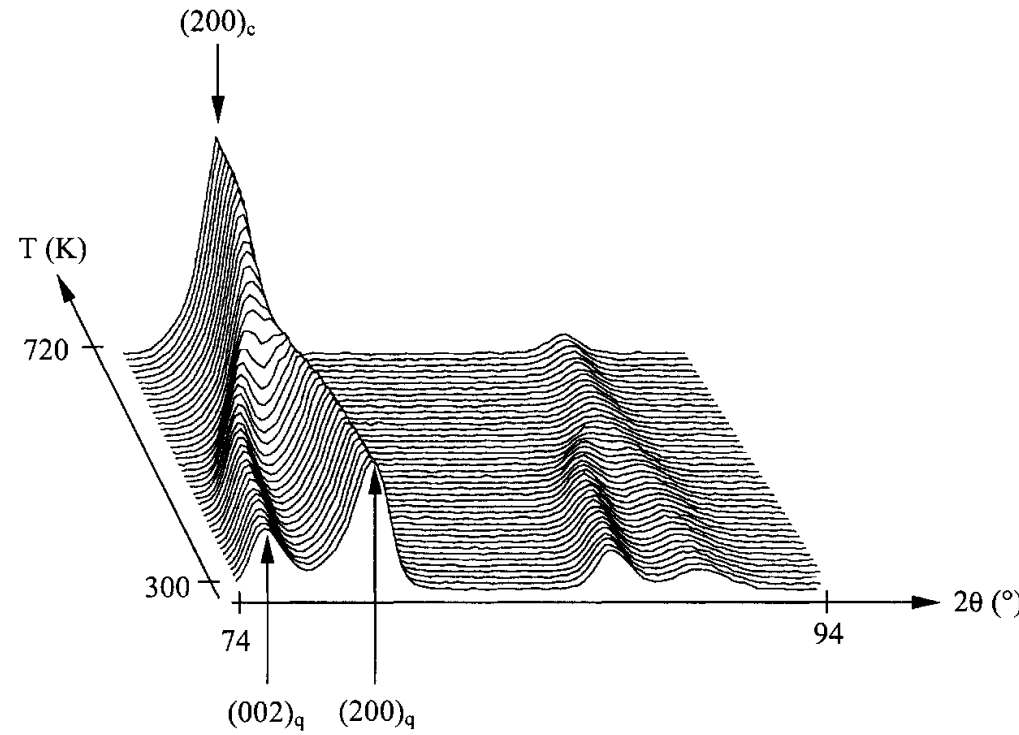

Figure 26: Représentation tridimensionnelle des diagrammes de diffraction enregistrés sur l'oxyde $\mathrm{PbHf}_{0,2} \mathrm{Ti}_{0,8} \mathrm{O}_{3}$ [31] .

Une étude détaillée des transitions de phase induites par la température a également été menée par diffraction de neutrons sur poudre sur l'oxyde $\mathrm{PbHf}_{0,8} \mathrm{Ti}_{0,2} \mathrm{O}_{3}$ [32]. Ce composé présente deux 
transitions de phase : la première apparaît vers $415 \mathrm{~K}$ entre deux phases ferroélectriques rhomboédriques notées $F_{R L}$ et $F_{R H}$ (groupes d'espace $R 3 c$ et $R 3 m$ respectivement), et la seconde, détectée vers $525 \mathrm{~K}$, sépare la phase $F_{R H}$ de la phase paraélectrique cubique $P_{C}$ (groupe d'espace $P m \overline{3} m$ ). Un paramètre d'ordre a été défini pour chacune de deux transitions : le paramètre d'ordre associé à la transition ferroélectrique--ferroélectrique est l'angle de rotation $\omega$ des octaèdres des atomes d'oxygène autour de l'axe ferroélectrique ; celui associé à la transition ferroélectriqueparaélectrique est la polarisation spontanée. Une étude par diffraction de neutrons à haute résolution (instrument 3T2 au LLB) a permis de calculer les cartes de densités nucléaires des atomes d'oxygène mettant en évidence la rotation des octaèdres autour de l'axe ferroélectrique (Fig. 27a). D'autre part, une étude par thermodiffractométrie des neutrons sur poudres a permis de suivre in situ la séquence de transitions de phase (instrument D1B à l'ILL, $\lambda=2,5 \AA$ ). La Figure $27 \mathrm{~b}$ présente l'évolution de l'angle de rotation $\omega$ en fonction de la température. Une approche phénoménologique de champ effectif a permis de rendre compte des variations expérimentales du paramètre d'ordre de rotation des octaèdres et de conclure à l'existence d'une transition ferroélectrique-ferroélectrique du $2^{\text {nd }}$ ordre (courbe calculée sur la Figure 27b).

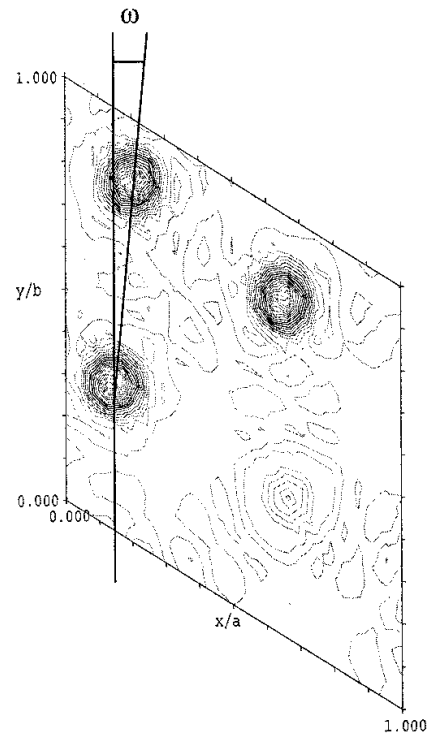

a)

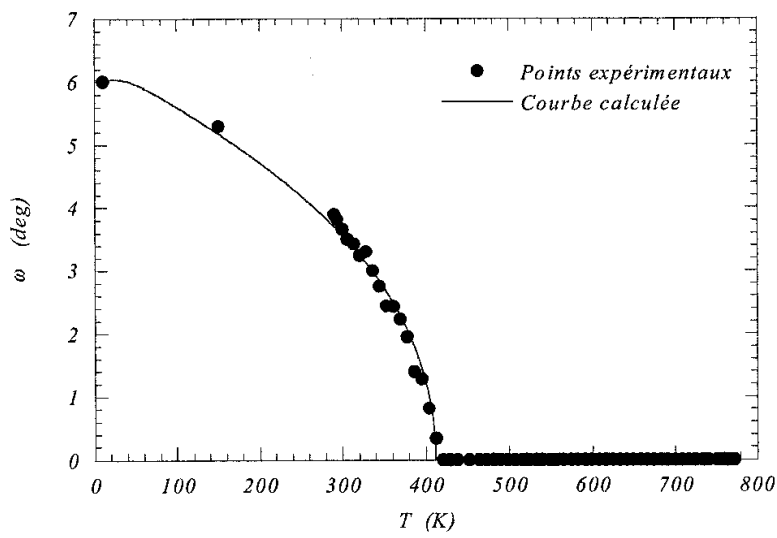

b)

Figure 27 : (a) Cartes de densités nucléaires des atomes d'oxygène mettant en évidence la rotation des octaèdres autour de l'axe ferroélectrique.

(b) Evolution thermique de l'angle de rotation des octaèdres. La courbe en trait plein a été calculée en utilisant une théorie phénoménologique [32].

\subsubsection{Equilibres solide-solide et solide-liquide dans un alliage ternaire}

Des travaux récents ont porté sur l'exploration du diagramme de phase ternaire $\mathrm{Al}-\mathrm{Zn}-\mathrm{Ga}$ dans lequel ont été mis en évidence des phénomènes inédits associés à une miscibilité rétrograde du gallium dans la solution solide $\mathrm{Al}-\mathrm{Zn}$ [33]. Ces phénomènes conduisent notamment à une inversion de cristallisation-fusion : un alliage partiellement liquide cristallise au chauffage puis fond partiellement au refroidissement. En compléments des techniques expérimentales classiques (ATD, DSC, dilatométrie, diffraction des rayons $\mathrm{X}, \ldots$ ), une étude de diffusion de neutrons a été menée sur l'alliage ternaire $\mathrm{Al}_{29,2} \mathrm{Ga}_{27} \mathrm{Zn}_{43,8}$ afin d'analyser les différentes transformations de phases [34]. L'avantage principal des neutrons dans ce cas est de permettre la réalisation d'une étude sur un 
échantillon massif (plusieurs $\mathrm{cm}^{3}$ ) afin de s'affranchir des effets de surface problématiques en diffraction des rayons $\mathrm{X}$.

Les expériences ont été effectuées sur l'instrument 7C2 au LLB. Le multidétecteur de 640 cellules et la courte longueur d'onde incidente $(\lambda=0,705 \AA)$ font de $7 \mathrm{C} 2$ un instrument très bien adapté à l'étude des liquides ou des matériaux amorphes (les caractéristiques de 7C2 sont proches de celles de D4 à l'ILL (cf. §5.3)). Les données de diffraction ont été enregistrées sur un domaine en $Q$ allant de 0,5 à $16 \AA^{-1}$.

L'alliage $\mathrm{Al}_{29,2} \mathrm{Ga}_{27} \mathrm{Zn}_{43,8}$ a été étudié de la température ambiante à $760 \mathrm{~K}$. Sur cette gamme de température, cet alliage présente une succession de transformations de phase :

- $S S_{\alpha}+S S_{Z n}+L \rightarrow S S_{\alpha}+S S_{\alpha}+L$ à $396 \mathrm{~K}$;

- $S S_{\alpha}+S S_{\alpha^{\prime}}+L \rightarrow S S_{\alpha}+S S_{\alpha^{\prime}}$ à $448 \mathrm{~K}$;

- $S S_{\alpha}+S S_{\alpha^{\prime}} \rightarrow S S_{\alpha^{\prime}}$ à $519 \mathrm{~K}$;

- $S S_{\alpha^{\prime}} \rightarrow S S_{\alpha^{\prime}}+L$ à $592 \mathrm{~K}$;

- $S S_{\alpha^{\prime}}+L \rightarrow L$ à $731 \mathrm{~K}$.

où $S S_{\alpha}, S S_{\alpha}$, et $S S_{Z n}$ sont des solutions solides et $L$ est un liquide. La solution solide $S S_{\alpha}$ cristallise dans une structure cubique à faces centrées (groupe d'espace $F m \overline{3} m$ ) alors que la phase $S S_{a}$, est de symétrie rhomboédrique (groupe d'espace $R \overline{3} \mathrm{~m}$ ). Enfin, la solution solide $S S_{Z n}$ cristallise dans une structure hexagonale compacte (groupe d'espace $P 6_{3} / \mathrm{mmc}$ ).

Les expériences menées sur $7 \mathrm{C} 2$ ont permis de suivre en fonction de la température les équilibres solide-liquide et solide-solide. Toutefois, la très faible résolution instrumentale de ce diffractomètre ne permet pas de séparer les deux solutions solides $S S_{\alpha}$ et $S S_{\alpha}$ ' très proches structuralement. La Figure 28 présente le diagramme de diffraction à $370 \mathrm{~K}$ et la diffusion diffuse mesurée à $760 \mathrm{~K}$ lorsque l'alliage est complètement fondu (représentation en échelle logarithmique).

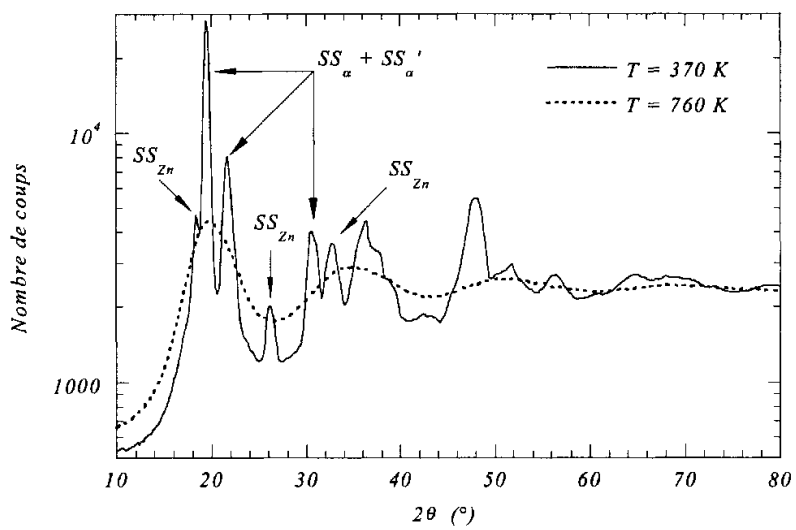

Figure 28: Diagrammes de diffraction enregistré à 370 et $760 \mathrm{~K}$ sur l'alliage $\mathrm{Al}_{29,2} \mathrm{Ga}_{27} \mathrm{Zn}_{43.8}$ [34].

L'étude des phases solides, à partir de la méthode de Rietveld, a permis de confirmer les différentes transformations et de révéler des phénomènes d'orientation préférentielle très importants après passage dans la phase liquide $(\mathrm{T}>731 \mathrm{~K})$. D'autre part, l'analyse des modulations du bruit de fond diffus a permis de mettre en évidence, par simulation du facteur de structure à partir d'un modèle de clusters, un ordre local icosaédrique lorsque l'alliage est complètement fondu [35].

Des études complémentaires sont en cours afin de déterminer les compositions chimiques des phases $S S_{\alpha}$ et $S S_{\alpha}$, et leurs fractions massiques respectives lorsqu'elles coexistent. Pour cela, des 
diagrammes ont été enregistrés sur le diffractomètre $3 \mathrm{~T} 2$ (LLB), la haute résolution de cet instrument permettant de séparer sans ambiguïté les contributions des phases $S S_{\alpha}$ et $S S_{\alpha^{\prime}}$ (Fig. 29).

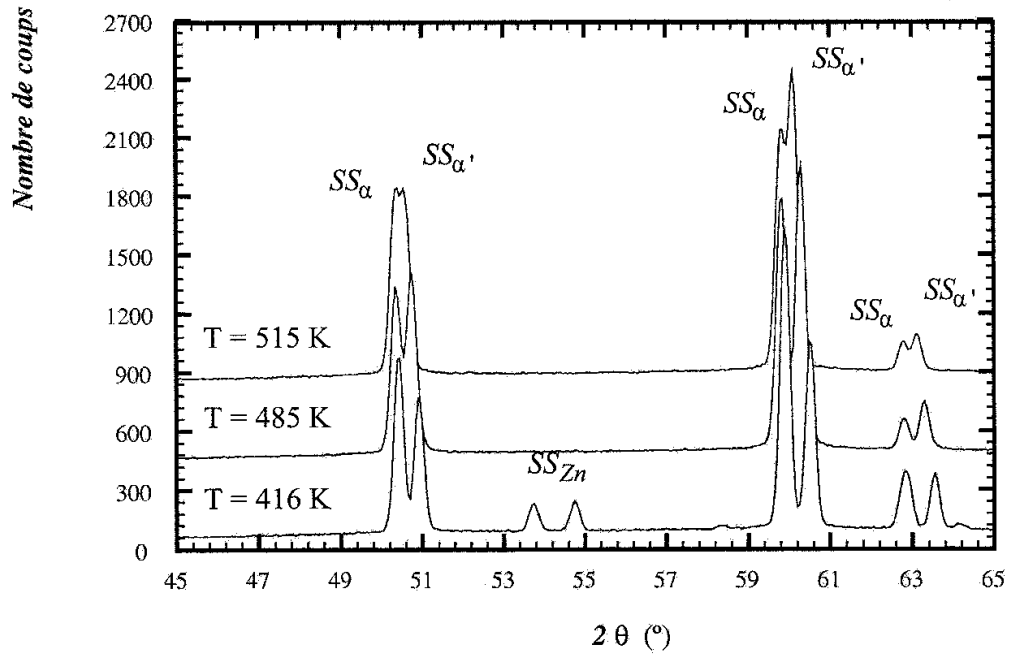

Figure 29: Evolution thermique des diagrammes enregistrés sur le diffractomètre haute résolution 3T2 (LLB).

\subsubsection{Transitions magnétiques étudiées par diffraction de neutrons sur poudres}

Les perovskites de formule générale $\mathrm{R}_{1-\mathrm{x}} \mathrm{A}_{\mathrm{x}} \mathrm{MnO}_{3}$ (où $\mathrm{R}$ est une terre rare et $\mathrm{A}$ un alcalin) présentent un grand intérêt du fait de la grande variété de leurs propriétés physiques. La plupart des études effectuées sur ce type de composés vise à corréler la modification des propriétés magnétiques et électroniques aux changements structuraux.

Lees et al. ont examiné, par diffraction de neutrons sur poudres, l'évolution des propriétés magnétiques et structurales de la série $\mathrm{Pr}_{0,6}\left(\mathrm{Ca}_{1-\mathrm{x}} \mathrm{Sr}_{\mathrm{x}}\right)_{0,4} \mathrm{MnO}_{3}$, en fonction de la température et de la composition $x$ [36]. Une première expérience de diffraction de neutrons a été effectuée sur le diffractomètre D1B à l'ILL $(\lambda=2,5 . \AA)$, les diagrammes de diffraction étant collectés de manière séquentielle entre 1,6 et $320 \mathrm{~K}$. La Figure 30 présente l'évolution thermique des diagrammes enregistrés sur les échantillons $\mathrm{Pr}_{0,6} \mathrm{Ca}_{0,4} \mathrm{MnO}_{3}$ (Fig. 30a) et $\mathrm{Pr}_{0,6}\left(\mathrm{Ca}_{0,75} \mathrm{Sr}_{0,25}\right)_{0,4} \mathrm{MnO}_{3}$ (Fig. 30b).

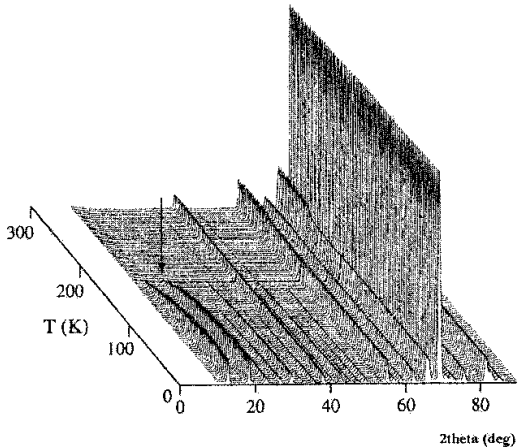

a)

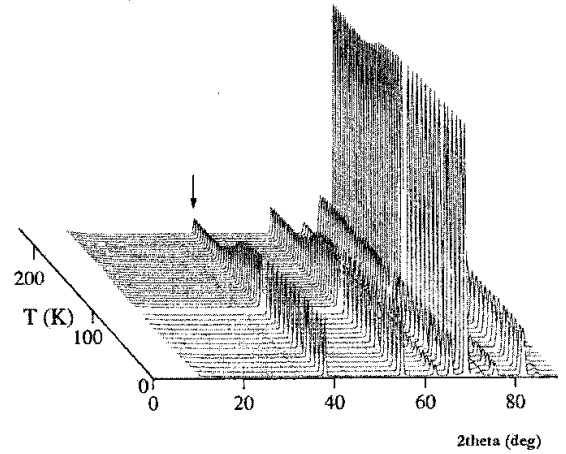

b)

Figure 30: Représentation tridimensionnelle des diagrammes de diffraction enregistrés en fonction de la température sur les échantillons (a) $\mathrm{Pr}_{0,6} \mathrm{Ca}_{0,4} \mathrm{MnO}_{3}$ (ordre antiferromagnétique) et (b) $\mathrm{Pr}_{0,6}\left(\mathrm{Ca}_{0,75} \mathrm{Sr}_{0,25}\right)_{0,4} \mathrm{MnO}_{3}$ (ordre ferromagnétique) [36]. 
La Figure 30 atteste que le comportement magnétique de cette série de composés est très fortement corrélé à la composition chimique $x$. En effet, le composé $\mathrm{Pr}_{0,6} \mathrm{Ca}_{0,4} \mathrm{MnO}_{3}$ présente un ordre antiferromagnétique en-dessous d'une température de Néel de $170 \mathrm{~K}$, alors que l'oxyde $\mathrm{Pr}_{0,6}\left(\mathrm{Ca}_{0,75} \mathrm{Sr}_{0,25}\right)_{0,4} \mathrm{MnO}_{3}$ présente quant à lui un ordre ferromagnétique en dessous de $150 \mathrm{~K}$. Une étude systématique de plusieurs compositions a permis de montrer que ces oxydes sont ferromagnétiques dès que $x \geq 0,2$, la température de transition augmentant très vite avec $x$.

Dans un second temps, connaissant les températures de transition, des diagrammes de bonne qualité ont été enregistrés sur le diffractomètre haute résolution D2B à l'ILL $(\lambda=1,594 \AA)$ afin de réaliser une analyse fine des structures magnétiques et nucléaires des composés de cette série [36].

\subsubsection{Exemple d'un diffractomètre sur source à spallation}

L'instrument HRPD est un diffractomètre de neutrons sur poudres installé sur la source à spallation ISIS en Grande-Bretagne (Rutherford Appelton Laboratory). Comme cela a déjà été mentionné dans le paragraphe 3.1, une source à spallation délivre des "pulses" de neutrons polychromatiques. La méthode dite de temps de vol assure la discrimination des longueurs d'onde à partir de la mesure du temps que mettent les neutrons pour atteindre le détecteur. Cette méthode permet ainsi de mesurer différentes distances interréticulaires pour un angle de diffusion fixé (cf. §3.1). La relation qui lie le temps de vol $t_{h k l}$ (exprimé en $\mu s$ ) à la distance interréticulaire $d_{h k l}$ (exprimée en $\AA$ ) est la suivante :

$$
t_{h k l}=505,55685 . L \cdot d_{h k l} \cdot \sin \theta
$$

où $L$ représente la distance parcourue par les neutrons et $2 \theta$ l'angle de diffusion. Pour une distance $L$ de $100 \mathrm{~m}$ et une distance interréticulaire de $1 \AA$, le temps de vol est de l'ordre de $50 \mathrm{~ms}$.

Le diffractomètre HRPD est configuré de façon à ce que les neutrons soient détectés dans l'une des trois banques de détecteurs de position fixée. Trois positions sont généralement utilisées : 20 proche de $180^{\circ}$ (diffusion en retour ou backscattering), $2 \theta=90^{\circ}$ et petits angles $\left(20^{\circ} \leq 2 \theta \leq 30^{\circ}\right.$ ), sachant que la résolution instrumentale optimale est obtenue en mode backscattering (Fig. 31). Le problème de recouvrement des pulses de neutrons (les neutrons les plus rapides d'un pulse peuvent arriver avant les neutrons lents du pulse précédent) est évité en utilisant deux choppers (cf. §3.2.4).
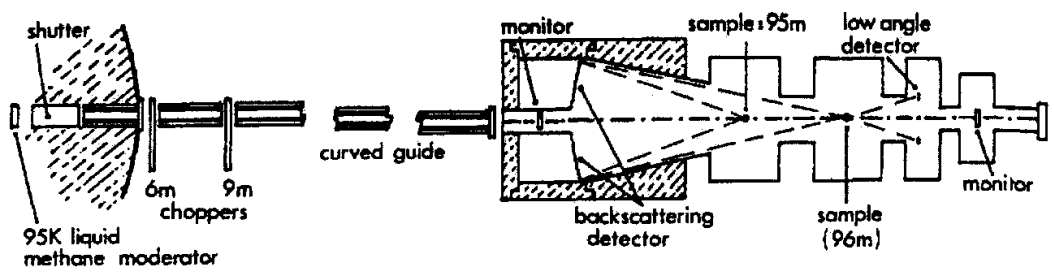

Figure 31: Schéma de principe d'un diffractomètre "temps de vol".

La diffraction des neutrons sur poudres est très souvent utilisées pour la détermination de structures de cristaux moléculaires. A titre d'illustration, Ibberson et al. ont déterminé la structure du méta-xylène deutéré $\mathrm{C}_{8} \mathrm{D}_{10}$ à l'aide de diagrammes de diffraction sur poudres enregistrés sur le diffractomètre HRPD à 4,5 K [37]. Les paramètres de maille ont été déterminés à l'aide d'une méthode d'indexation automatique et le groupe d'espace a été déduit des extinctions systématiques (maille orthorhombique : $a=16,830 \AA, b=10,492 \AA$ et $c=7,582 \AA$ et groupe d'espace $P b c a$ ). La structure a ensuite été résolue par une méthode de recuit simulé puis vérifiée en utilisant les méthodes directes à partir d'une collecte 4-cercles rayons X. La Figure 32a compare les diagrammes "temps de vol" observé et calculé. Le Figure $32 \mathrm{~b}$ présente le dessin de la molécule à $4,5 \mathrm{~K}$. 


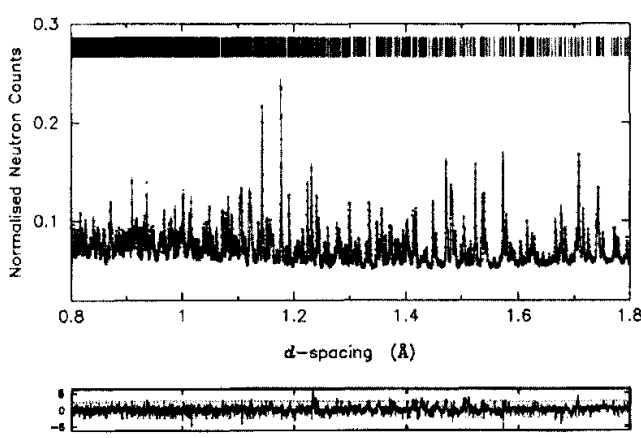

a)

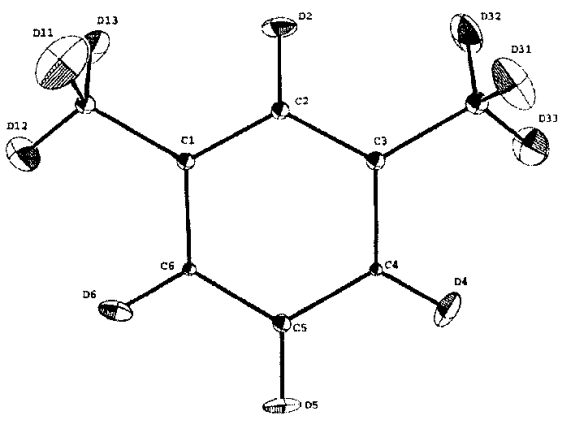

b)

Figure 32: (a) Diagramme "temps de vol" observé, calculé et différence [37]

(b) Dessin de la molécule méta-xylène à $4,5 \mathrm{~K}$ (les ellipsoïdes de vibration sont représentés pour le deutérium).

\section{CONCLUSION}

Que l'on effectue une expérience de diffraction de neutrons sur monocristal ou sur poudre, les informations cristallographiques essentielles auxquelles on peut avoir accès sont la position moyenne des noyaux des atomes de la structure, leurs paramètres de déplacements atomiques et l'arrangement des moments magnétiques dans le cas de structures magnétiques. Cependant, la diffraction sur poudres présente certains inconvénients comparativement à la diffraction sur monocristal, la principale limitation résidant dans le recouvrement angulaire des réflexions de Bragg lié en particulier à la réduction à une dimension des trois dimensions de l'espace réciproque. Il peut donc être délicat de déterminer précisément la contribution de chaque réflexion sur un domaine angulaire donné. Ceci explique certainement que la diffraction sur monocristal a longtemps été préférée à la diffraction sur poudres. Toutefois, l'amélioration de la résolution instrumentale des diffractomètres associée au développement intensif des méthodes d'ajustement de profil font actuellement de la diffraction sur poudres une technique puissante, facile de mise en ceuvre et très bien adaptée aux études in situ et temps réel. Il en résulte donc que le choix entre "diffraction sur monocristal" et "diffraction sur poudre" dépend essentiellement de la problématique sous-tendue par l'étude et de la possibilité d'obtention de monocristaux de taille suffisante.

Quelle que soit la technique de diffraction finalement choisie, il est toujours nécessaire de connaitre les caractéristiques instrumentales (monochromateur, collimateur, filtres...) du diffractomètre afin de trouver le compromis longueur d'onde - flux - résolution instrumentale le mieux adapté à l'étude du composé.

\section{Ouvrages et documents de base}

Neutron and synchrotron radiation for condensed matter studies, HERCULES, Les Editions de la Physique, Les Ulis (1993).

- R. Scherm and B. Fåk, Neutrons, Hercules Vol. I, p. 113.

- G.J. McIntyre, Single crystal diffractometry with monochromatic X-rays and neutron, Hercules Vol. I, p. 179.

- J. Pannetier, Powder diffraction techniques, Hercules Vol. I, p. 207.

- V.T. Forsyth, Fibre diffraction, Hercules Vol. I, p. 247.

- G. Heger, Single crystal structure analysis, Hercules Vol. П, p. 23.

- J. Schweizer, Neutron scattering and magnetic structures, Hercules Vol. II, p. 97.

J. Pannetier, Techniques d'analyse des diagrammes de diffraction des rayons $X$ et des neutrons par les poudres, Formation Permanente du CNRS (1995).

M. Anne, Utilisation des différents types d'instruments en science des matériaux, Ecole de Printemps "Diffusion des Neutrons et Science des Matériaux" (1997).

http://www.ill.fr/Info/diff_neutrons/Programme/Programme.htm 


\section{Références}

1. S. Janssen, D. Rubio-Temprano, A. Furrer, Time versus monochromatic focusing on a cold neutron time of flight spectrometer, Physica B 283, 355 (2000).

2. P. Mikula, J. Kulda, P. Lukas, M. Ono, M. Vrana, V. Wagner, Instrumentation components of focusing diffraction used in NPI, ILL, KURRI and PTB, Physica B 276-278, 174 (2000).

3. H.J. Bleif, D. Wechsler, F. Mezei, TOF powder diffractometer on a reactor source, Physica B 276-278, 181 (2000).

4. L.D. Cussen, A design for improved neutron collimators, Nucl. Instrum. Meth. Phys. Res. A 414, 365 (1998).

5. Th. Kirst, F. Mezei, High-performance, short solid state collimators with reflecting walls, Nucl. Instrum. Meth. Phys. Res. A 450, 389 (2000).

6. C. Petrillo, F. Sachetti, O. Toker and N.J. Rhodes, Solid state neutron detectors, Nucl. Instrum. Meth. Phys. Res. A 378, 541 (1996).

7. C. Petrillo, F. Sachetti, G. Maehlum, M. Mancinelli, Space resolution of Si/Gd microstrip as linear position sensitive detector for thermal neutrons, Nucl. Instrum. Meth. Phys. Res. A 424, $523(1999)$.

8. P. Convert, M. Berneron, R. Gandelli, T. Hansen, A. Oed, A. Rambaud, J. Ratel, J. Torregrossa, A large counting rate one-dimensional position sensitive detector: the D20 banana, Physica B 234-236, 1082 (1997).

9. P. Convert, T. Hansen, A. Oed, J. Torregrossa, D20 high-flux two-axis neutron diffractometer, Physica B 241-243, 195 (1998).

10. P. Fischer, G. Frey, M. Koch, M. Könnecke, V. Pomjakushin, J. Schefer, R. Thut, N. Schlumpf, R. Bürge, U. Greuter, S. Bondt, E. Berruyer, High-resolution powder diffractometer HRPT for thermal neutrons at SINQ, Physica B 276-278, 146 (2000).

11. M. Marmotti, M. Haese-Seiller, R. Kampmann, Two-dimensional position-sensitive ${ }^{3} \mathrm{He}-$ neutron detector for reflectometry and high-resolution diffractometry, Physica B 276-278, 210 (2000).

12. C. Wilkinson, P. Schobinger-Papamantellos, D. Myles, L.D. Tung, K.H.J. Buschow, On the modulated structure of $\mathrm{La}_{2} \mathrm{Co}_{1.7}$ : a neutron Laue diffraction study, J. Magn. Magn. Mater. 217, $55(2000)$.

13. A.J. Peurrung, Recent developments in neutron detection, Nucl, Instrum. Meth. Phys. Res. A $443,400(2000)$.

14. S. Tazaki, K. Neriishi, K. Takahashi, M. Etoh, Y. Karasawa, S. Kumazawa, N. Niimura, Development of a new type of imaging plate for neutron detection, Nucl. Instrum. Meth. Phys. Res. A 424, 20 (1999).

15. G. Caglioti, A. Paoletti, F.P. Ricci, Choice of collimators for a crystal spectrometer for neutron diffraction, Nucl. Instrum. 3, 223 (1958).

16. A. Hewat, Design for conventional high resolution neutron powder diffractometer, Nucl. Instrum. Meth. 127, 361 (1975).

17. Ch. Muller, M. Anne, M. Bacmann, M. Bonnet, Structural studies of the fast oxygen ion conductor BICOVOX.15 at room temperature, J. Solid State Chem. 141, 241 (1998).

18. Ch. Muller, Transition ordre-désordre et conductivité anionique dans les composés $\mathrm{Bi}_{4}\left(V_{I-x} M e_{x}\right)_{2} \mathrm{O}_{11-\delta}, \mathrm{Me}=\mathrm{Co}, \mathrm{Fe}$, Thèse, Université J. Fourier - Grenoble I (1996).

19. S. Obbade, P. Wolfers, D. Fruchart, R. Argoud, J. Muller, E. Palacios, A precise crystal structure determination. Part II: An X-ray four-circle study of $\mathrm{Nd}_{2} \mathrm{Fe}_{14} \mathrm{~B}$ at 20 and $290 \mathrm{~K}$, J. Alloys Comp. 242(1-2), 80 (1996).

20. P. Wolfers, S. Obbade, D. Fruchart, R. Verhoef, Precise crystal and magnetic structure determinations. Part I: a neutron diffraction study of $\mathrm{Nd}_{2} \mathrm{Fe}_{14} \mathrm{~B}$ at $20 \mathrm{~K}$, J. Alloys Comp. 242(1-2), 74 (1996).

21. D.N.H. Nam, L.D. Tung, P. Nordblad, N.P. Thuy, N.X. Phuc, Magnetic anomalies of polycrystalline $\mathrm{La}_{2} \mathrm{Co}_{1.7}$ compound in the temperature range $5-400 \mathrm{~K}$, J. Magn. Magn. Mater. 177-181, 1135 (1998).

22. P. Langan, Neutron diffraction fibre: recent advances at $I L L$, Physica B 234-236, 213 (1997).

23. Y. Nishiyama, T. Okano, P. Langan, H. Chanzy, High resolution neutron fibre diffraction data on hydrogenated and deuterated cellulose, Int. J. Biological Macromolecules 26, 279 (1999). 
24. J. Schefer, M. Könnecke, A. Murasik, A. Czopnik, Th. Strässle, P. Keller, N. Schlumpf, N., Single-crystal diffraction instrument TriCS at SINQ, Physica B 276-278, 168 (2000).

25. A. Murasik, A. Czopnik, L. Keller, P. Fischer, Spin rotation in $E r G a_{3}$, J. Magn. Magn. Mater. 213(1-2), 101 (2000).

26. M. Anne, Ab initio structure determination with $X$-ray and neutron powder diffraction data, Formation Permanente du CNRS (1992).

27. H.M. Rietveld, Line profiles of neutron powder diffraction peaks for structure refinement, Acta Cryst. 22, 151 (1967).

28. H.M. Rietveld, A profile refinement method for nuclear and magnetic structures, J. Appl. Cryst. 2, 65 (1969).

29. J.I. Langford, D. Louër, Powder diffraction, Reports Prog. Phys. 59, 131 (1996).

30. L.B. McCusker, R.B. Von Dreele, D.E. Cox, D. Louër, P. Scardi , Rietveld refinement guidelines, J. Appl. Cryst. 32, 36 (1999).

31. C. Bedoya, Ch. Muller, J-L. Baudour, F. Bouree, J-L. Soubeyroux, M. Roubin, Ferroelectricparaelectric phase transition in $\mathrm{PbHf}_{0.2} \mathrm{Ti}_{0.8} \mathrm{O}_{3}$ studied by neutron powder diffraction, J. Phys.: Condens. Matter 13(30), 6453 (2001).

32. Ch. Muller, J-L. Baudour, C. Bedoya, F. Bouree, J-L. Soubeyroux, M. Roubin, Octahedral deformations and cationic displacements in the ferroelectric $\mathrm{PbHf}_{0.8} \mathrm{Ti}_{0.2} \mathrm{O}_{3}$ : a neutron powder diffraction study from 10 to $770 \mathrm{~K}$, Acta Cryst. B56, 27 (2000).

33. Aragon, E., Jardet, K., Satre, P. and Sebaoun, A., Al-Zn-Ga phase diagram, J. Therm. Anal. 53, 769 (1998).

34. K. Jardet, Ch. Muller, R. Bellissent, P. Satre, A. Sebaoun, Temperature dependent X-ray and neutron diffraction study of the liquid-solid and solid-solid equilibria in the $\mathrm{Al}_{29.2} \mathrm{Ga}_{27} \mathrm{Zn}_{43.8}$ ternary alloy, J. All. Comp. 316(2), 179 (2001).

35. K. Jardet, Contribution à l'étude du diagramme de phases du système Al-Zn-Ga. Détermination d'un point critique de miscibilité à l'état solide et d'un point d'évanouissement de la ligne monovariante de liquidus correspondante, Thèse, Université de Toulon et du Var (1998).

36. M.R. Lees, J. Barratt, G. Balakrishnan, D.McK. Paul, C. Ritter, Neutron powder diffraction study of the magnetic and structural properties of $\mathrm{Pr}_{0,6}\left(\mathrm{Ca}_{1-x} \mathrm{Sr}_{x}\right)_{0,4} \mathrm{MnO}_{3}(0 \leq x \leq 1)$, Phys. Rev. B 58(13), 8694 (1998).

37. R.M. Ibberson, W.I.F. David, S. Parsons, M. Prager, K. Shankland, The crystal structures of $m$-xylene and p-xylene, $C_{8} D_{10}$, at $4.5 \mathrm{~K}$, J. Mol. Structure 524(1-3), 121 (2000).

\section{Sites Internet}

Caractéristiques des instruments de l'Institut Laue Langevin (ILL) : http://www.ill.fr/YellowBook/ Caractéristiques des instruments du Laboratoire Léon Brillouin (LLB) : http://wwwllb.cea.fr/spectros/spectros.html

Source à spallation ISIS (Rutherford Appelton Laboratory) : http://www.isis.rl.ac.uk/ Swiss Spallation Neutron Source (SINQ) : http://www1.psi.ch/www_sinq_hn/Welcome_SINQ.html "Teaching Pamphlets" de l'IUCr : http://www.ch.iucr.org/iucr-top/comm/cteach/pamphlets.html 